\title{
RAC1 activation drives pathologic interactions between the epidermis and immune cells
}

\author{
Mårten C.G. Winge, ${ }^{1}$ Bungo Ohyama, ${ }^{1,2}$ Clara N. Dey, ${ }^{1}$ Lisa M. Boxer, ${ }^{1}$ Wei Li, ${ }^{1}$ Nazanin Ehsani-Chimeh, ${ }^{1}$ Allison K. Truong, ${ }^{1}$ \\ Diane Wu, ${ }^{1}$ April W. Armstrong, ${ }^{3}$ Teruhiko Makino, ${ }^{1}$ Matthew Davidson, ${ }^{4}$ Daniela Starcevic, ${ }^{1}$ Andreas Kislat, ${ }^{5}$ Ngon T. Nguyen, ${ }^{1}$ \\ Takashi Hashimoto, ${ }^{2}$ Bernard Homey, ${ }^{5}$ Paul A. Khavari, ${ }^{1}$ Maria Bradley, ${ }^{6}$ Elizabeth A. Waterman, ${ }^{1}$ and M. Peter Marinkovich ${ }^{1,7}$ \\ 1Program in Epithelial Biology, Stanford University School of Medicine, Stanford, California, USA. ²Department of Dermatology, Kurume University School of Medicine, Kurume, Japan. \\ ${ }^{3}$ Department of Dermatology, Keck School of Medicine at the University of Southern California, Los Angeles, California, USA. ${ }^{4}$ Department of Immunology and Rheumatology, \\ Stanford University School of Medicine, Stanford, California, USA. 'D Department of Dermatology, Medical Faculty, Heinrich-Heine University, Dusseldorf, Germany. \\ ${ }^{6}$ Department of Dermatology, Karolinska Institutet, Stockholm, Sweden. ${ }^{7}$ Dermatology Service, Veterans Affairs Medical Center, Palo Alto, California, USA.
}

\begin{abstract}
Interactions between the epidermis and the immune system govern epidermal tissue homeostasis. These epidermis-immune interactions are altered in the inflammatory disease psoriasis; however, the pathways that underlie this aberrant immune response are not well understood. Here, we determined that Ras-related C3 botulinum toxin substrate 1 (RAC1) is a key mediator of epidermal dysfunction. RAC1 activation was consistently elevated in psoriatic epidermis and primary psoriatic human keratinocytes (PHKCs) exposed to psoriasis-related stimuli, but not in skin from patients with basal or squamous cell carcinoma. Expression of a constitutively active form of RAC1 (RAC ${ }^{\mathrm{V} 12}$ ) in mice resulted in the development of lesions similar to those of human psoriasis that required the presence of an intact immune system. RAC1 ${ }^{\text {V12 }}$-expressing mice and human psoriatic skin showed similar RAC1-dependent signaling as well as transcriptional overlap of differentially expressed epidermal and immune pathways. Coculture of PHKCs with immunocytes resulted in the upregulation of RAC1-dependent proinflammatory cytokines, an effect that was reproduced by overexpressing RAC1 in normal human keratinocytes. In keratinocytes, modulating RAC1 activity altered differentiation, proliferation, and inflammatory pathways, including STAT3, NFKB, and zinc finger protein 750 (ZNF750). Finally, RAC1 inhibition in xenografts composed of human PHKCs and immunocytes abolished psoriasiform hyperplasia and inflammation in vivo. These studies implicate RAC1 as a potential therapeutic target for psoriasis and as a key orchestrator of pathologic epidermis-immune interactions.
\end{abstract}

\section{Introduction}

Interactions between cutaneous epithelia and the immune system are vital for maintaining epidermal tissue homeostasis, skin barrier integrity, and immunocyte responses (1). Disruption of this interplay can lead to psoriasis, a chronic inflammatory disorder affecting $3 \%$ of all individuals (2). Psoriasis is characterized by pruritic, disfiguring skin lesions, arthritis in up to $30 \%$ of cases (3), and increased risk of myocardial infarction and stroke (4).

Immune target identification has led to new immune-based biologic therapies. However, potential risks of systemic immunosuppressive therapy such as serious infections (5) and tuberculosis activation (6), along with a lack of a full understanding of the long-term biologic immunosuppressive risks (7), make the search for alternatives to long-term systemic immunosuppression highly relevant, especially in the treatment of a lifelong disorder such as psoriasis.

Despite the longstanding recognition of epidermal dysfunction as an intrinsic feature of psoriasis and the implication of epidermal pathways in the analysis of psoriasis genome-wide association studies (8), significant gaps still exist in our understanding

Conflict of interest: The authors have declared that no conflict of interest exists. Submitted: November 30, 2015; Accepted: April 27, 2016.

Reference information: / Clin Invest. 2016;126(7):2661-2677. doi:10.1172/JCI85738. of the role of the epidermis in psoriasis pathogenesis (9) and have limited the development of epidermis-targeted therapies.

One aspect of the disease process that has confounded mechanistic studies to date is the observation that psoriasis is not only genetic in origin, but is also highly responsive to various environmental stimuli, with cutaneous wounding, known as the Koebner phenomenon, (10) and group A streptococcal (GAS) infection being the two most well-known psoriasis triggers (11). The question of how seemingly distinct environmental triggers can interact with predisposing genetic factors to activate psoriasis has not been answered, and a unifying model incorporating both genetic and environmental influences in psoriasis pathogenesis has not yet been established. However, one potential clue lies in the epidermal activation of the small GTPase Rasrelated $\mathrm{C} 3$ botulinum toxin substrate 1 (RAC1). RAC1 activation is known to be triggered in response to extracellular environmental signals (12), a process believed to promote the epidermal proliferation required for wound repair (13). Furthermore, the binding of streptococcal capsular polysaccharide with the keratinocyte CD44 cell-surface receptor strongly induces epidermal RAC1 activation (14). These findings, which appear to link together diverse external psoriasis triggers, led us to examine the role of epidermal RAC1 activation as a possible etiologic factor in psoriasis pathogenesis. 
A

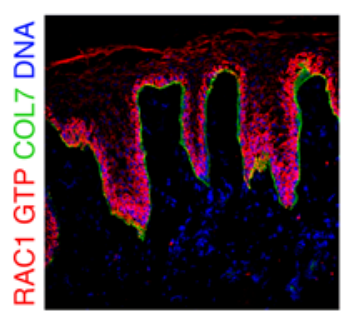

C

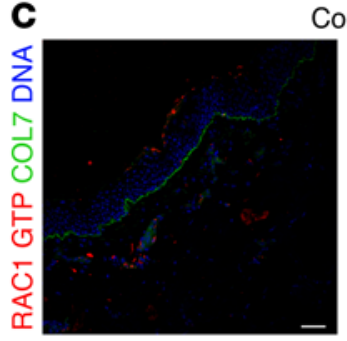

Control

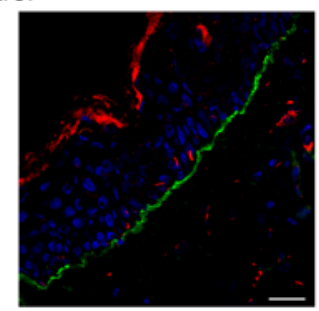

F

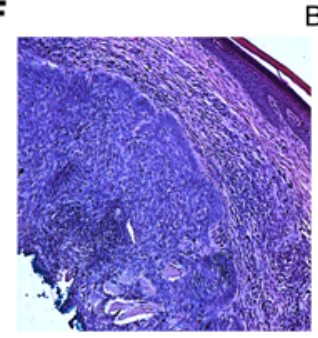

BCC

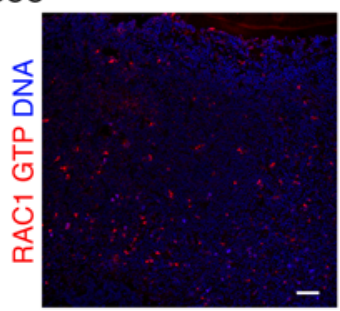

B
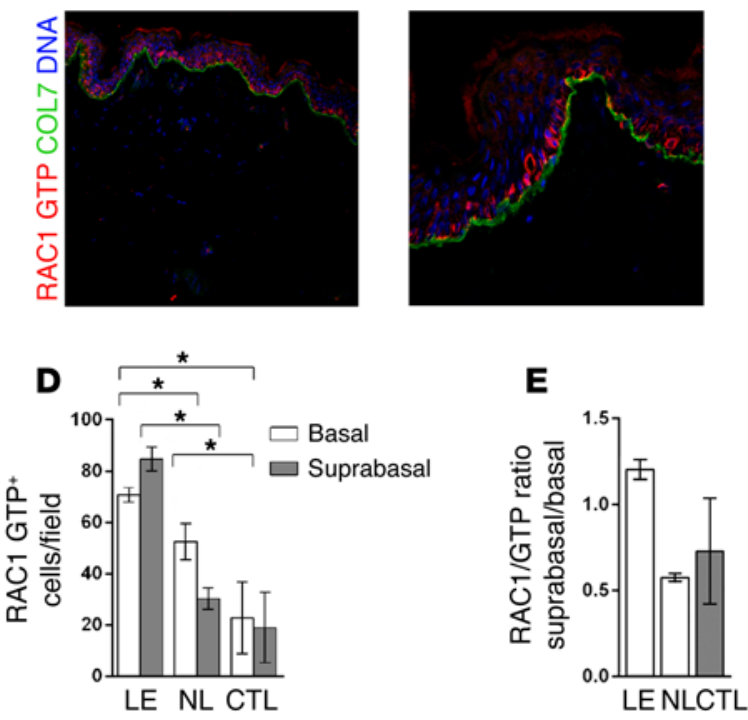

G

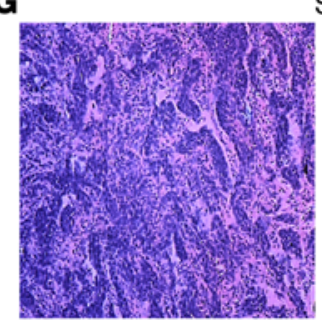

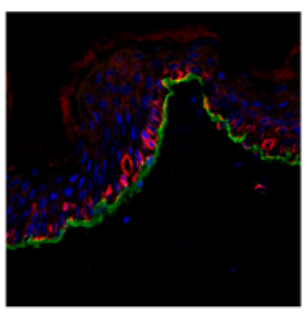

SCC

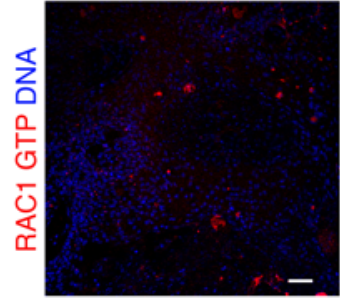

H
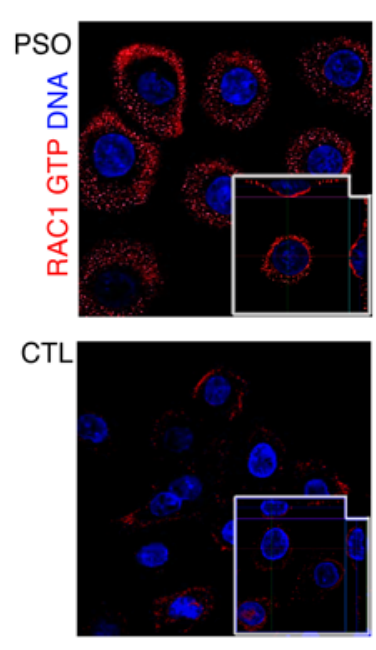

V12

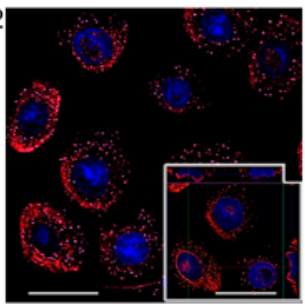

I EGF RAC1 GTP pulldown

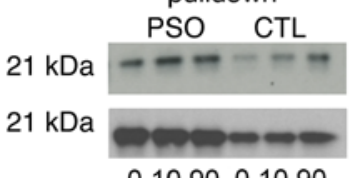

0109001090

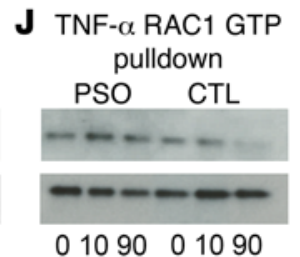

M

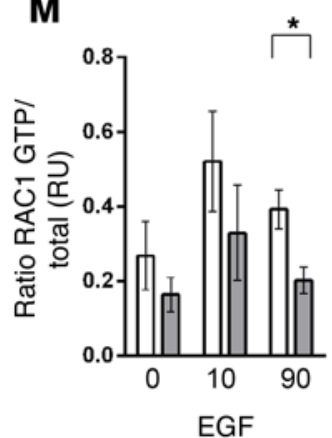

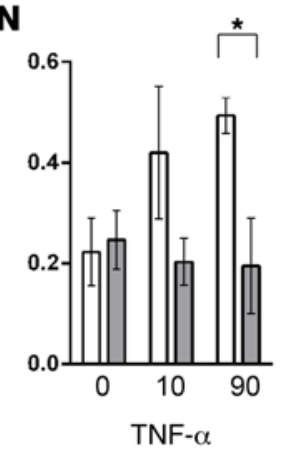

pulldown

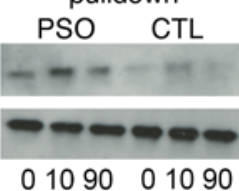

L IL-22 RAC1 GTP pulldown

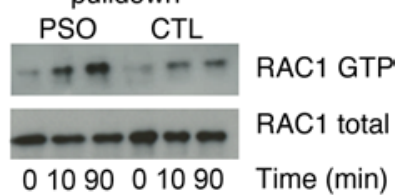

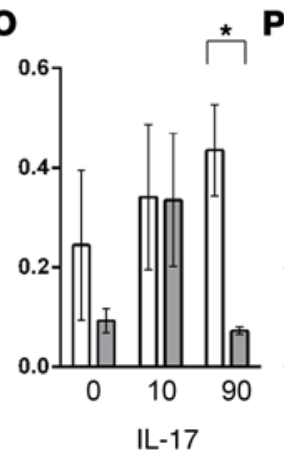

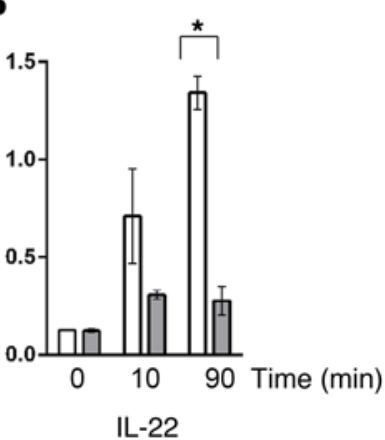


Figure 1. An epidermal intrinsic defect of RAC1 hyperactivation in human psoriasis. (A) Human psoriatic lesional, (B) nonlesional, and (C) control skin and (F) basal and (C) squamous cell carcinomas (BCC and SCC) were examined by IDIF confocal microscopy using a RAC1 GTP-specific mAb. RAC1 GTP, red; type VII collagen, green. (D and E) RAC1 GTP was significantly elevated in psoriatic lesional suprabasal and basal epidermis and in basal nonlesional psoriatic epidermis compared with control skin. (H) PHKCs $(n=7)$ and RAC1 ${ }^{V 12}$ keratinocytes showed elevated cytosolic RAC1 activation compared with NHKCs by confocal microscopy. RAC1 GTP, red; DNA, blue. ( $\mathbf{I}$ and $\mathbf{M})$ EGF, ( $\mathbf{J}$ and $\mathbf{N}$ ) TNF- $\alpha$, (K and $\mathbf{0})$ IL-17A/F, and ( $\mathbf{L}$ and $\mathbf{P}$ ) IL-22 triggered RAC1 hyperactivation 90 minutes after cytokine stimulation, assayed by RAC1 GTP pulldown. Error bars represent SEM. (D and $\mathbf{M}-\mathbf{P}){ }^{*} P<0.05$, unpaired $t$ test. $\mathbf{A}, n=19 ; \mathbf{B}, n=4 ; \mathbf{C}, n=10 ; \mathbf{F}$ and $\mathbf{G}$, $n=3 ; \mathbf{H}, n=7$ (PSO), $n=4$ (CTL/V12). $\mathbf{D}$ and $\mathbf{E}, n=19$ (LE), $n=4$ (NL), $n=10$ (CTL). I and $\mathbf{M}, n=4 ; \boldsymbol{J}, \mathbf{K}, \mathbf{N}$, and $\mathbf{O} n=3 ; \mathbf{L}$ and $\mathbf{P}, n=2$ per condition. PSO, PHKCs; CTL, NHKCs; V12, RAC1 ${ }^{\mathrm{V} 12}$-overexpressing NHKCs; COL7, type VII collagen. Scale bars: $50 \mu \mathrm{m}$ (A-C, left, F, and C); $25 \mu \mathrm{m}$ (A-C, right); $20 \mu \mathrm{m}$ (H). LE, lesional psoriatic skin; NL, nonlesional psoriatic skin.

\section{Results}

Epidermal RAC1 hyperactivation in human psoriasis. To examine the activation state of RAC1, psoriatic skin was tested by indirect immunofluorescence (IDIF) microscopy using a RAC1-GTP-specific mAb. Abnormally high RAC1 activation was observed (Figure 1, A-E, and Supplemental Figure 1A; supplemental material available online with this article; doi:10.1172/JCI85738DS1) in suprabasal and basal lesional epidermis $(n=19)$ and in basal nonlesional epidermis $(n=4)$. However, RAC1 activation was not elevated in basal $(n=3)$ or squamous cell $(n=3)$ carcinoma, or in a mouse contact dermatitis model (Figure 1, F and G, and Supplemental Figure 1C). In contrast, another Rho GTPase, RhoA, showed no increased activation in psoriatic skin (Supplemental Figure 1B). The specificity of these Abs was confirmed using organotypic 3D skin equivalents with keratinocytes overexpressing activated mutants of RAC1 (RAC1 $\left.1^{V 12}\right)$, RhoA (RHOA $\left.{ }^{V 14}\right)$, or $L a c Z$ control (Supplemental Figure 2).

To further investigate the significance of epidermal RAC1 activation in psoriasis, primary psoriatic human keratinocytes (PHKCs) from nonlesional skin, cultured in serum-free medium $(n=7)$, showed marked, cytoplasmic RAC1-GTP distribution compared with a reduced, peripheral distribution in primary normal human keratinocytes (NHKCs) ( $n=4$; Figure $1 \mathrm{H})$. NHKCs overexpressing an activated (V12) RAC1 mutant (12) (RAC1 ${ }^{\text {V12 }}$ NHKCs) showed an intracellular distribution similar to that of PHKCs. Following growth factor starvation, the addition of the psoriasisrelated stimuli EGF, TNF- $\alpha$, IL-17A/F, IL-22, or GAS capsular extract (but not IL-6, data not shown) triggered RAC1 hyperactivation in PHKCs compared with that seen in NHKCs (Figure 1, I-P; Supplemental Figure 3, A and B; and Supplemental Figure 4A). In total, all PHKCs tested consistently showed marked RAC1 hyperactivation in response to exogenous stimuli.

Epidermal RAC1 hyperactivation in mice closely mimics human psoriasis. To determine the role of epidermal RAC1 activation in psoriasis, a keratin 14-driven (K14-driven), V12-activated RAC1 mutant (ref. 15 and Figure 2A) was expressed in transgenic mice and validated by immunoblotting ( $\mathrm{RAC}^{\mathrm{V} 12}$ band, upper arrow, Figure 2B) and IDIF (Figure 2C). WT control skin showed minimal RAC1 activation, except focally at the wound edges (Supplemental Figure 6A). Erythematous, scaly skin lesions developed by day 7
(Figure 2D), thickened by day 14 (Figure 2E), and typically localized to ears, paws, tail, and snout (Figure 2, F-M). Rac1 ${ }^{\text {V12 }}$-expressing mice ( $R a c 1^{V 12}$ mice) were smaller than their WT siblings. Rac1 $1^{V 12}$ mouse lesions showed hemorrhage (Auspitz sign) following scale removal (Figure 2J). Rac1 ${ }^{V 12}$ mothers often bit their pups' whiskers and snout hair (possibly to decrease nursing-related itching). Mutant, but not normal, pups developed psoriasis-like lesions in response to this trauma, similar to the Koebner phenomenon (10) of human psoriasis (Figure 2, K and L). Skin lesions improved following topical corticosteroid treatment (Figure 2, M and N). By 1 month, most mice developed erythema and edema of the tail and paws; however, approximately $50 \%$ showed a pronounced mutilating arthropathy, with bony paw deformations (Figure 2, O-R) and/or partial tail autoamputation.

Lesional RAC1 skin showed pronounced psoriasiform hyperplasia, hypogranulosis, mixed inflammatory infiltrates (Figure 3, A and B), dilated vessels in dermal papillae (Figure 3C, arrows), and marked parakeratosis (Figure 3D). Wound-induced psoriasiform hyperplasia typically followed tail snipping for genotyping (Figure 3, E and F). Mucosa, but not perimucosal skin, was spared from proliferative or inflammatory changes, as demonstrated in anus (Figure 3, G and H) and eyelids (Figure 3, I and J, lower arrows). $R a c 1^{V 12}$ mice with joint involvement showed neutrophilic infiltrates near joint spaces (Figure 3, K and L, arrow). Nail changes ranged from mild to severe, with nail matrix showing psoriasiform hyperplasia (Figure 3, M and N, arrow).

Lesional $\mathrm{Rac1}^{\mathrm{V12}}$ skin showed increased $\mathrm{CD} 4^{+}$and $\mathrm{CD}^{+}$ lymphocytes, neutrophils, and DCs by flow cytometry (Figure $4 \mathrm{~A})$. Increased $\mathrm{CD}^{+}$lymphocytes expressing IL-17 and ROR $\gamma$, and $\mathrm{CD}^{\circ} 8^{+}$cells (Munro's micro abscesses) in the stratum corneum were noted by IDIF (Figure 4, B-D; Supplemental Figure 6B). Suprabasilar proliferation (by Ki67 staining) was prominent (Figure 4H and Supplemental Figure 6E). The Rac1 ${ }^{\text {V12 }}$ transgene produced a similar psoriatic phenotype across CBA (Figures 2 and 3), BALB/c, and C57BL/6 backgrounds (not shown), however backcrossing to a nonobese diabetic/severe combined immunodeficiency (NOD/SCID) strain lacking functional lymphocytes strikingly reduced epidermal thickness (Figure 4E) and proliferation (Figure $4 \mathrm{H}$ ) as well as tail and joint abnormalities, despite persistent RAC1 activity in NOD/SCID Rac1 ${ }^{\text {V12 }}$ skin (Figure 4F). Similarly, cyclosporin A treatment significantly reduced epidermal thickness, proliferation, and $\mathrm{T}$ cell infiltration (Supplemental Figure 6, C-H). In total, these results suggest that epidermal RAC1 activation closely mimics the cutaneous and rheumatologic phenotype of human psoriasis, but only in the presence of an intact immune system.

Transcriptional overlapping signature of $R a c 1^{V 12}$ murine skin in human psoriatic lesional skin. Transcriptional analysis of day-7 $R a c 1^{V 12}$ mouse skin compared with WT littermate skin identified $46 \%$ induced and $54 \%$ repressed differentially expressed genes (DEGs) that were ranked by significance or after FDR correction (Figure 5A and Supplemental Table 1). Enriched canonical pathways involved the role of IL-17A in psoriasis, the antigen presentation pathway, CD40 signaling, and altered $\mathrm{T}$ cell and $\mathrm{B}$ cell signaling in rheumatoid arthritis (Figure $5 \mathrm{~B}$ ). Biological functions annotated to dermatological diseases and conditions (psoriasis); inflammatory response (organ inflammation); cellular movement; 
A

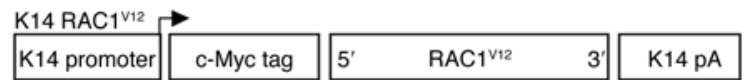

B
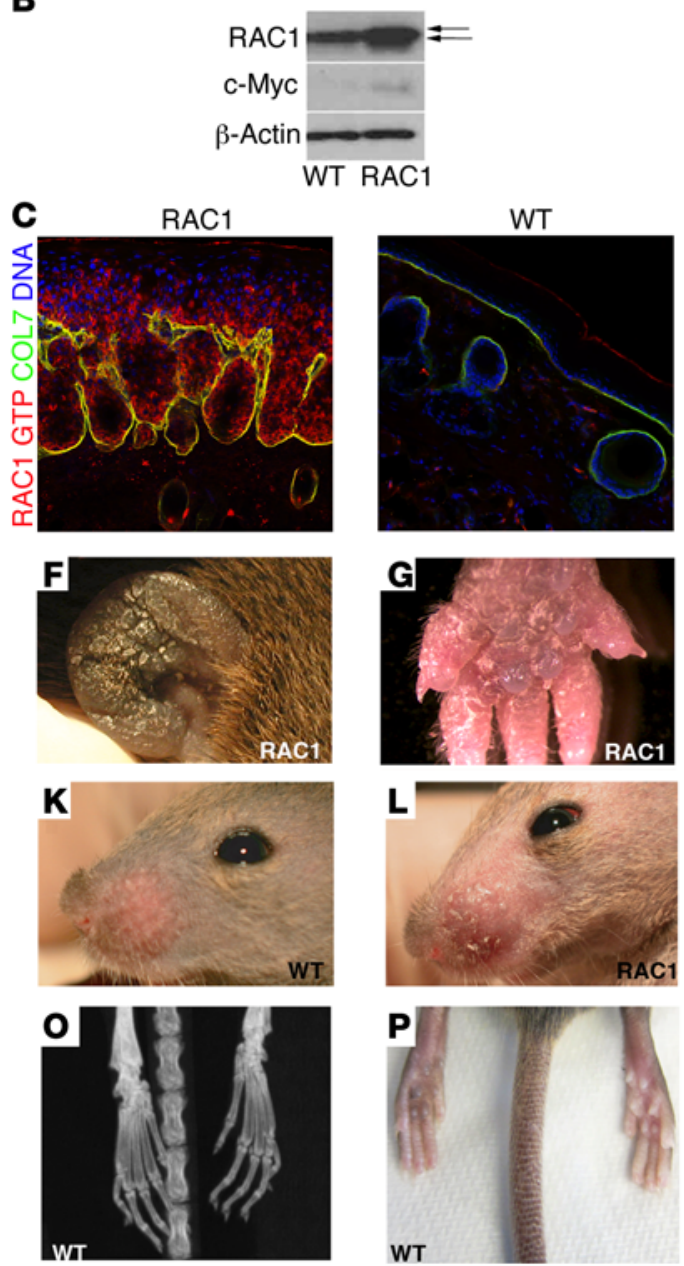
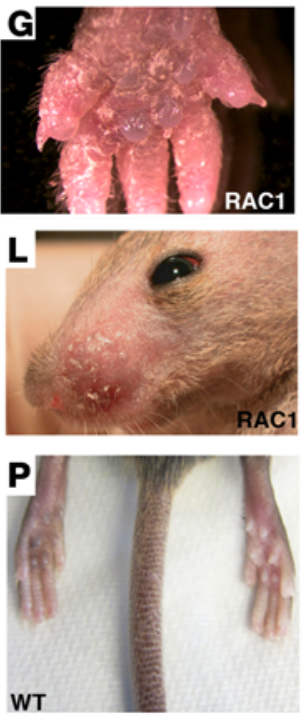

D
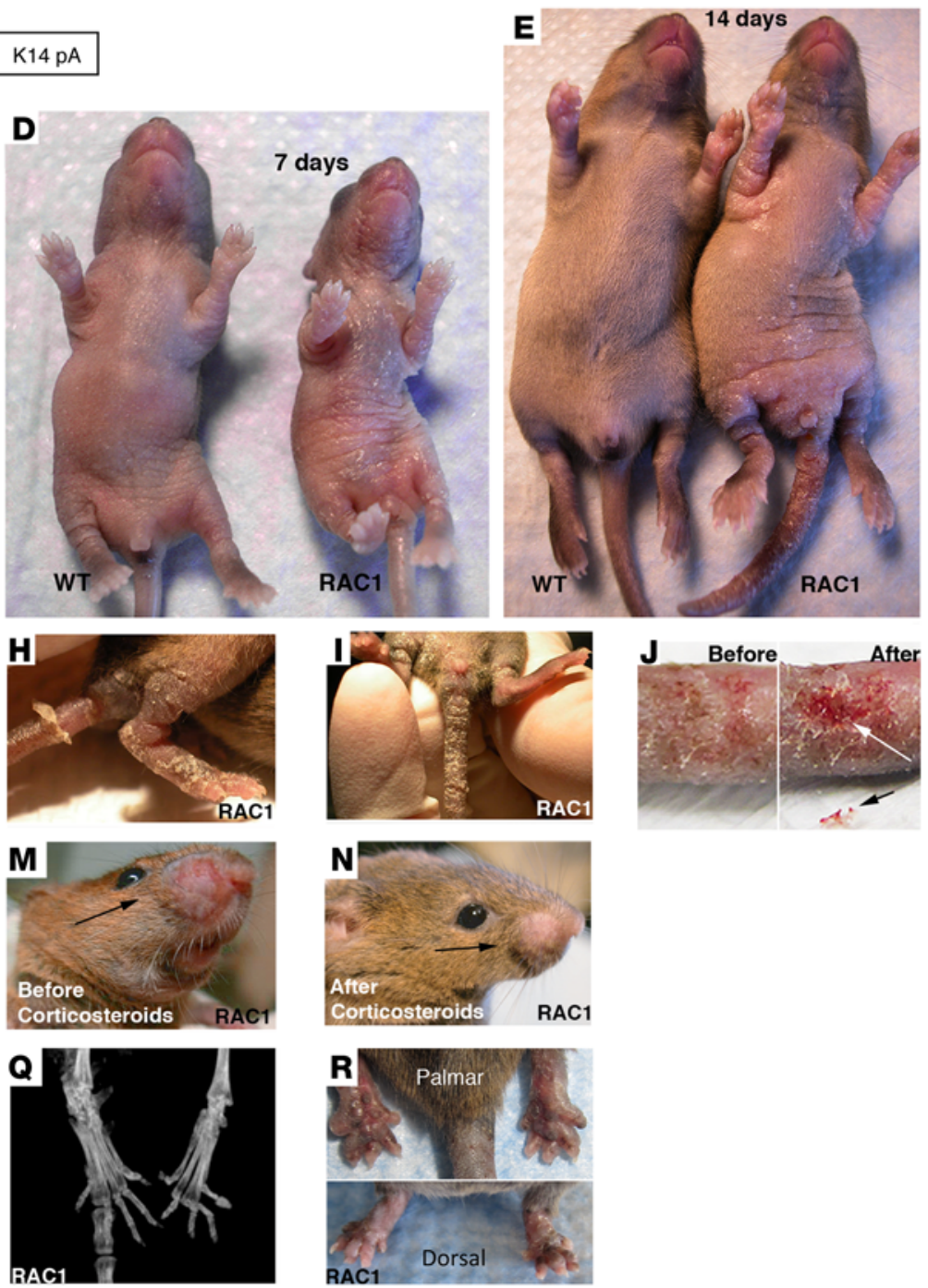
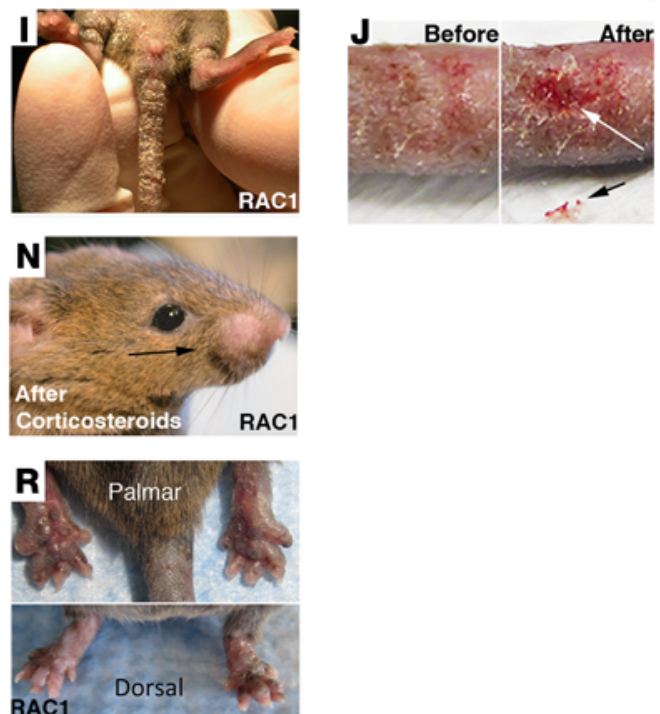

Figure 2. Epidermal RAC1 hyperactivation in mice closely mimics human psoriasis. (A) Generation of K14-driven, Rac $7^{1 / 2}$-activated mutant in transgenic mice demonstrating RAC1 hyperactivation by (B) immunoblot (Rac1 ${ }^{12}$ band, upper arrow) and (C) IDIF (original magnification, $\times 20$; RAC1 GTP, red; DNA, blue). (D) Localized skin erythema and scaling were apparent by day 7, (E) progressing by day 14. Rac112 mice were smaller compared with their WT siblings. (F-I) By 1 month, lesions typically localized to the ears, paws, tail, and snout. (J) Lesions showed hemorrhage (Auspitz sign) following removal of scales, and (K and $\mathbf{L}$ ) trauma-related lesion development resembled the Koebner phenomenon of human psoriasis. ( $\mathbf{M}$ and $\mathbf{N}$ ) Potent topical corticosteroids improved skin lesions. (O-R) By 1 month, erythema and edema of the tail and limbs around the distal joints and paws were frequent, and approximately $50 \%$ of mice showed a pronounced mutilating arthropathy, associated with bony deformations of the paws. (B and $\mathbf{C}$ ) $n=3$ per condition.

cellular growth and proliferation (proliferation of cells); organismal survival (organismal death); and embryonic development (formation of epidermis) (Figure 5C and Supplemental Table 2). Activation $z$-scores of transcription factors (TFs) and cytokines included STAT3, NFאB, IFN- $\gamma$, TNF- $\alpha$, IL-1 $\beta$, and IL-17A signaling (Figure 5, D and E). A human psoriasis data set yielded significant enrichment for IFN, STAT3, NFאB, and Rho family GTPase signaling (RAC1 but not RhoA) (Supplemental Figure 7A). Merging significance-ranked $\operatorname{Racl}^{V 12}$ mouse skin DEGs $(n=518)$ with this ranked human data set revealed a significantly overlapping signature $(n=139, P<0.05)$ (Figure $5 \mathrm{~F}$ ), including KRT16, S100A9, IL36A/RN, STAT3, and CGNL1 (Supplemental Table 3). Enriched pathways encompassed the role of IL-17A in psoriasis (IL17RC, S10OA8, and S100A9), agranulocyte adhesion and diapedesis, the role of cytokines in mediating communication between immu- nocytes, the protein ubiquitination pathway, and atherosclerosis signaling (Figure 5G). Biological functions included psoriasis, migration, proliferation, leukocyte homing, and psoriatic arthritis (Figure $5 \mathrm{H}$ ). Ingenuity pathway analysis TF motif enrichment included associations with JUN/FOS, STAT3, IFN regulatory factors 1,3 , and 7 (IRF1/3/7), and NFKB complex (REL/RELA/RELB/ NFkB1/NFkB2) (Supplemental Figure 7, B and C). Most of the significant nonoverlapping signatures in $R a c 1^{12}$ mouse skin included the antigen presentation pathway, retinoic acid receptor (RAR) activation, CD40 signaling, and atherosclerosis signaling (Supplemental Figure 7E), whereas most of the significantly induced nonoverlapping signatures in human psoriatic skin contained a protein ubiquitination pathway, cell-cycle regulation, molecular mechanisms of cancer, and IFN signaling (Supplemental Figure 7D). In silico mapping of psoriasis susceptibility genes to interac- 


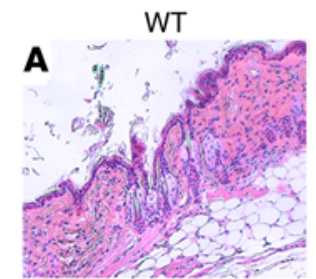

WT

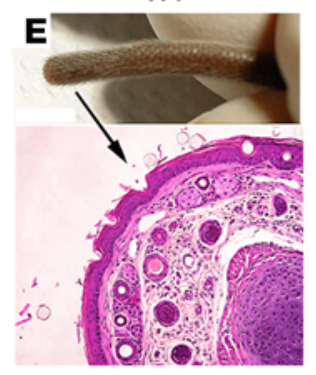
WT
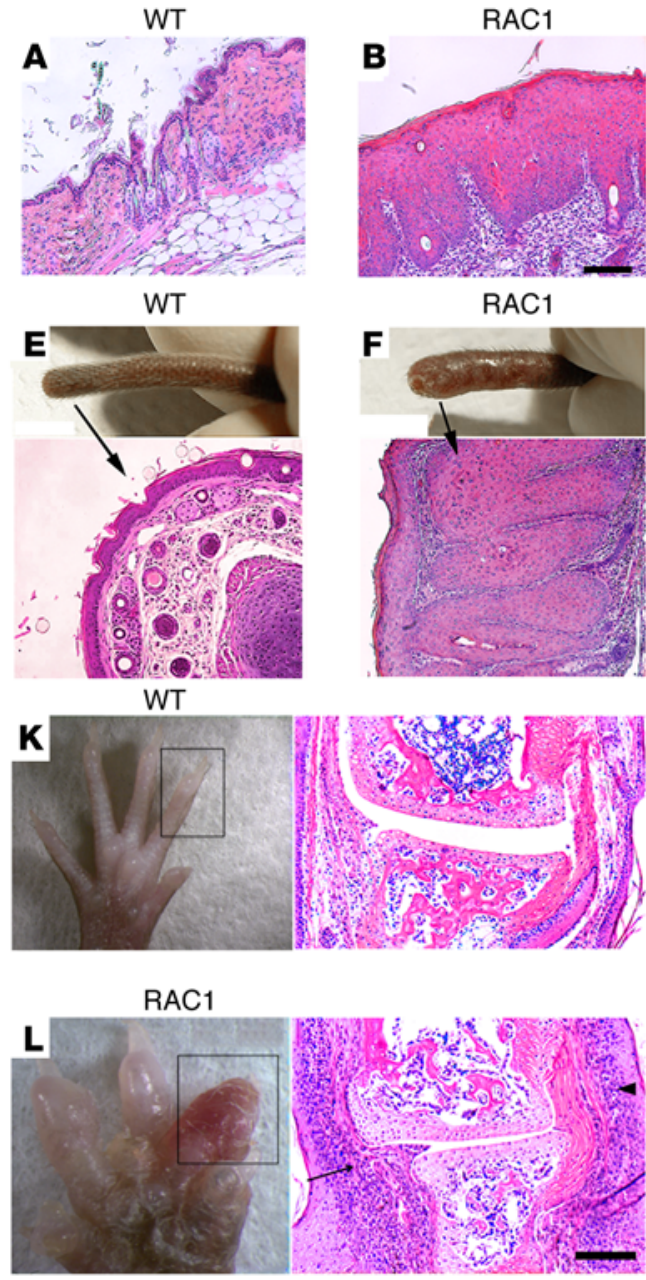

RAC1

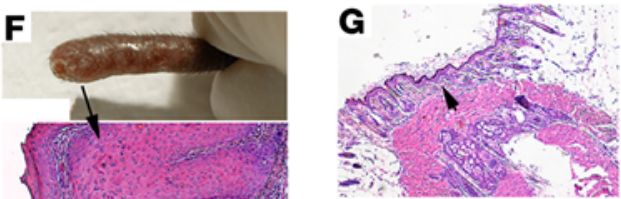

WT
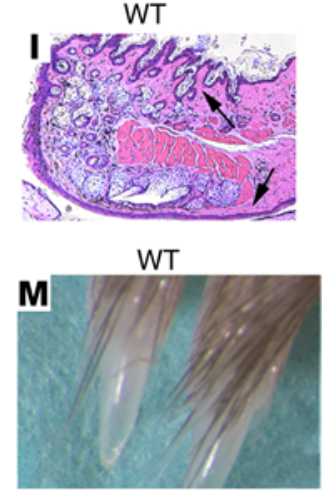

RAC1

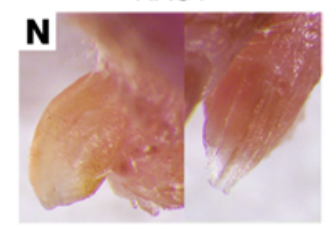

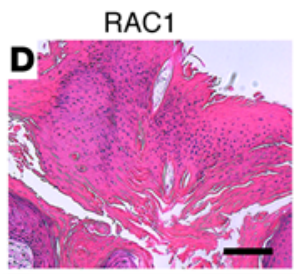

RAC1

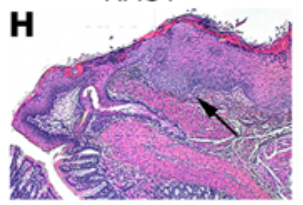

RAC1
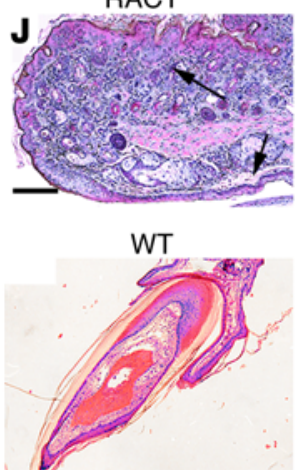

RAC1

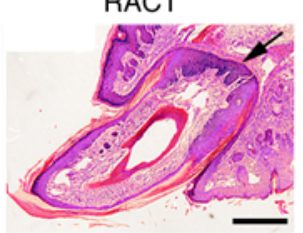

Figure 3. Epidermal RAC1 activation produces a psoriasiform phenotype including skin, nail, and joint changes. ( $A$ and $\mathbf{B}$ ) Lesional Rac1 ${ }^{112}$ skin showed psoriasiform hyperplasia, hypogranulosis, a mixed inflammatory infiltrate, (C, arrows) dilated vessels in dermal papillae, and (D) foci of marked parakeratosis. (E and $\mathbf{F}$ ) Wound-induced psoriasiform hyperplasia (Koebner phenomenon). ( $\mathbf{G}$ and $\mathbf{H}$ ) Perianal and (I and J, lower arrows) conjunctival mucosa (but not perimucosal skin) were spared from proliferative and inflammatory changes. (L, arrow) Rac1 $7^{12}$ mice with joint involvement showed neutrophilic infiltrates near joint spaces compared with WT controls (L compared with $\mathbf{K})$. ( $\mathbf{M}$ and $\mathbf{N}$, arrow) Nail changes ranged from ridging to marked thickening and onycholysis, and nail matrix showed psoriasiform hyperplasia. Scale bars: $200 \mu \mathrm{m}$ (M and $\mathbf{N}$ ), $100 \mu \mathrm{m}(\mathbf{A}-\mathbf{L})$. tions with each other and RAC1 implicated a network including JAK/STAT, NFאB, RAC1, and IRF signaling (Supplemental Figure $7 F$ ). Transcriptional overlap between $R a c 1^{V 12}$ and other mouse models of psoriasis (16) showed the most significant overlap with the K14AREG and K5STAT3C models (Supplemental Table 4). Enriched pathways included STAT3, immune cell, IL-22, and JAK signaling (Supplemental Figure $7 G$ ), whereas signatures enriched in $R a c 1^{V 12}$ skin included the antigen presentation pathway, the role of IL-17 in psoriasis, and atherosclerosis- and arthritis-associated pathways (Supplemental Figure 7H).

Rac1 ${ }^{12}$ murine skin mimics the proliferative, proinflammatory, and differentiation signaling seen in human psoriasis. Rac $1^{V 12}$ mouse and human psoriatic epidermis showed similar levels of proliferative and inflammatory protein expression. Mouse and human skin showed increased TGF- $\alpha$, CARD14 (17), and IL-23p19 (Figure $6, A-C)$, and $R a c 1^{V 12}$ skin showed increased mRNA expression of the psoriasis-associated $\beta$-defensins (18) Ccl17, Ccl2O, Ccl5, Ccr6 (19), as well asTslp (20), the oncostatin M receptor (Osmr) (21), and Il23p19 and Il1f6 (ref. 22 and Figure 6D). Rac1 ${ }^{\text {V12 }}$ skin showed activation of STAT3 and NFאB (Figure 7, A-D), which was verified by nuclear localization and immunoblot analysis (Figure 7, E-G); however, epidermal phosphorylated STAT3 (p-STAT3) was strongly reduced in epidermis of immunodeficient $\operatorname{Rac1}^{\text {V12 }}$ mice (Figure $7, \mathrm{H}$ and $\mathrm{I}$ ).
To assess the spatiotemporal relationships of psoriasis-associated chemokines in inflamed $\mathrm{Racl}^{\mathrm{V12}}$ skin, we compared epidermis and whole skin in 1-day-old $R a c 1^{V 12}$ and WT mice, preceding immune cell infiltration and psoriasiform hyperplasia (prelesional skin), with that of 7-day-old pup skin, when marked psoriasiform hyperplasia and immune cell infiltration were evident. We found a number of cytokine mRNAs, including Ccl2, Ccl5, Ccl2O, Cxcl1, 34 -defensin, Cxcl11, oncostatin $\mathrm{M}$ receptor (Osmr), and thymic stromal lymphopoietin (Tslp), that were significantly increased in prelesional epidermis (Supplemental Figure 8). By 1 week of age, there was a further increase in $\mathrm{Ccl} 2 \mathrm{O}$ and $I l 1 b$. Prelesional whole skin showed a marked increase in mRNA levels of cytokines such as Ccl2, Ccl5, Cxcl10, and Cxcl11, whereas lesional skin also included significantly upregulated mRNA levels of Cxcl2 and Il1b (Supplemental Figure 8). Because of the marked joint inflammation in $R a c 1^{v 12}$ mice, we tested whether RAC1 activation in the skin could be associated with induction of a systemic inflammatory response. A Luminex assay of mouse sera revealed that 3-week-old Rac1 ${ }^{\text {V12 }}$ mouse sera contained significant increases in the psoriasis-associated cytokine IL-22 ( 7-fold) and increases in IL-23 ( 2.5-fold) compared with levels detected in WT mouse sera (Figure 6E).

RAC1 activation promotes proliferation- and inflammationrelated signaling in PHKCs and xenografts. We next validated psoriasis-related proliferation and inflammation regulators involving 
A

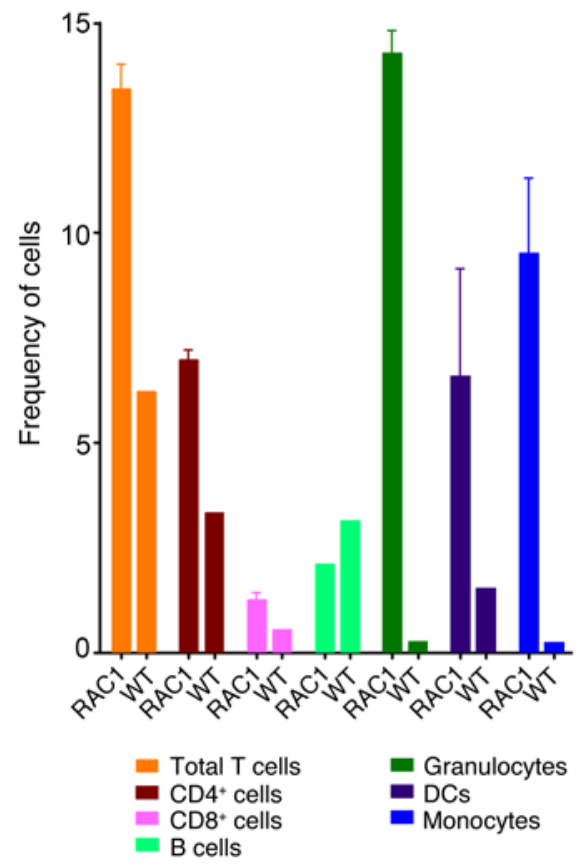

B

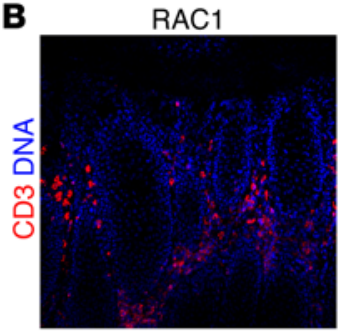

C

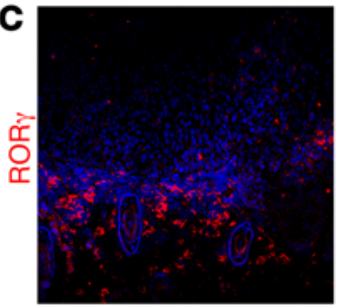

D

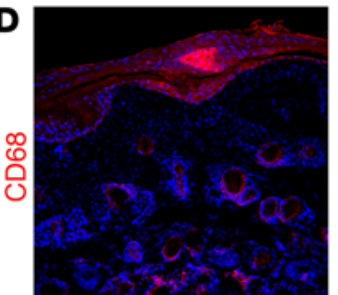

F RAC1 GTP

E
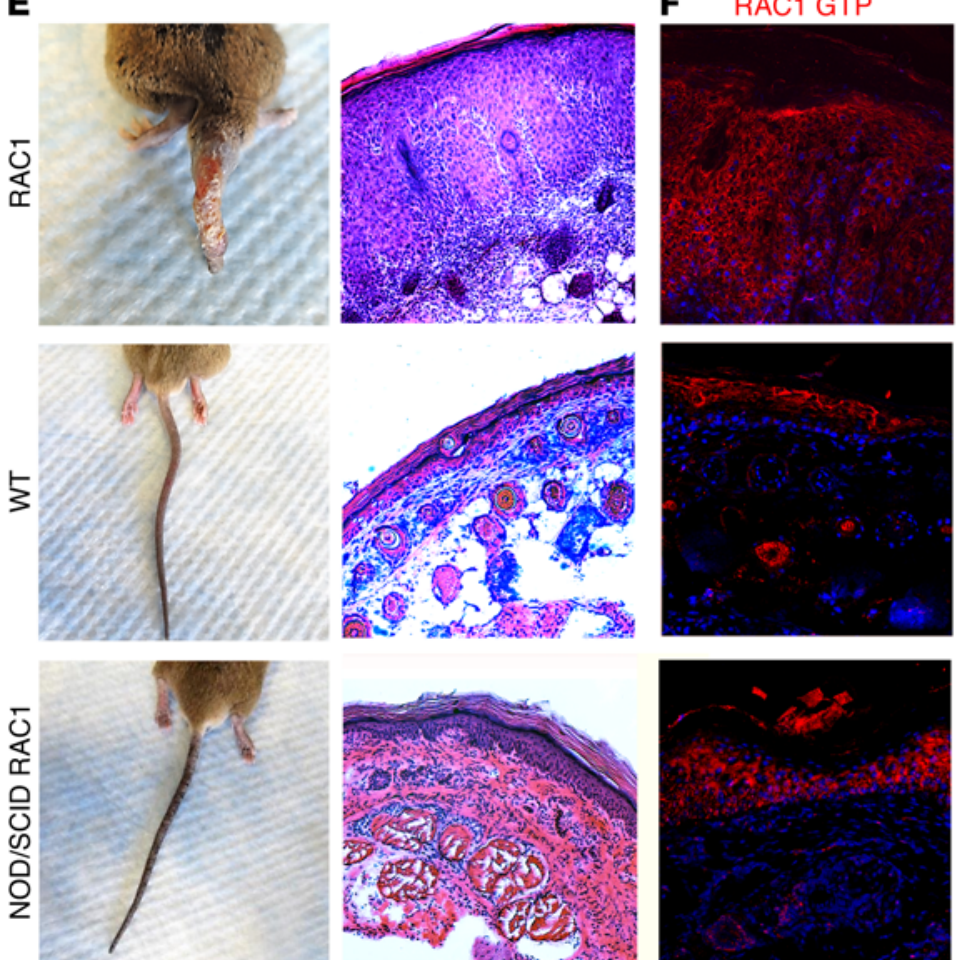

\section{G RhoA GTP}
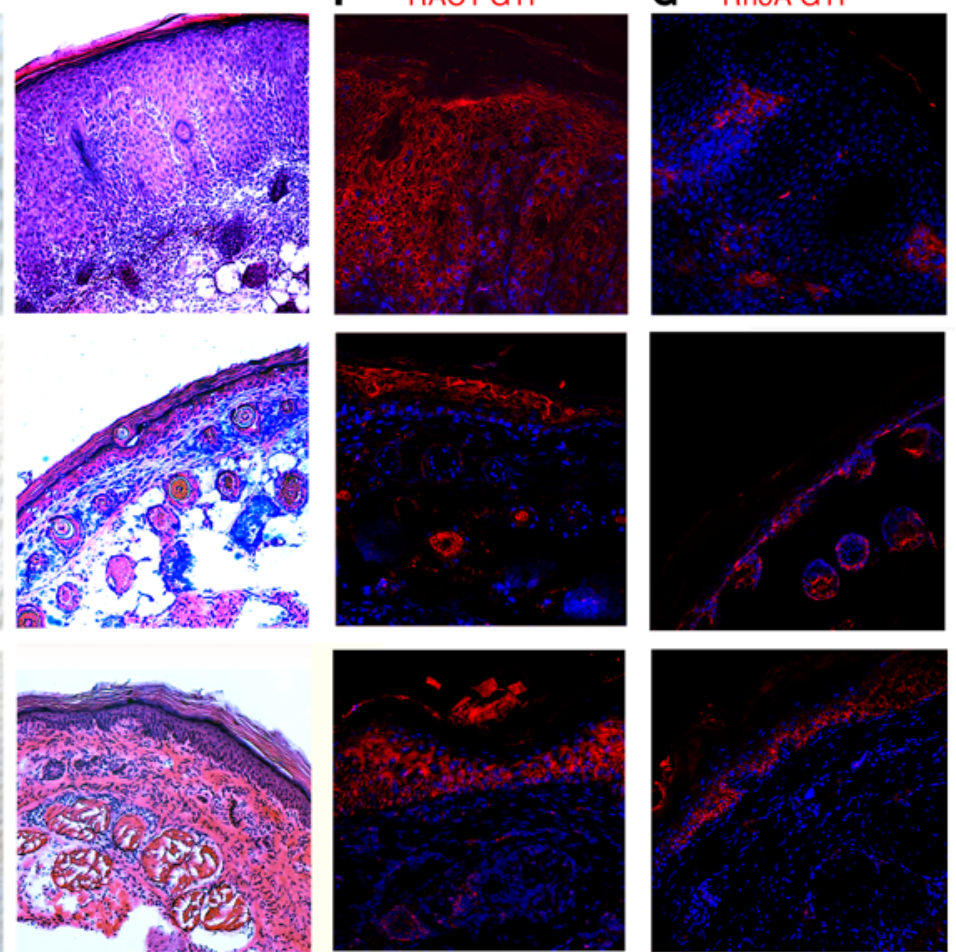
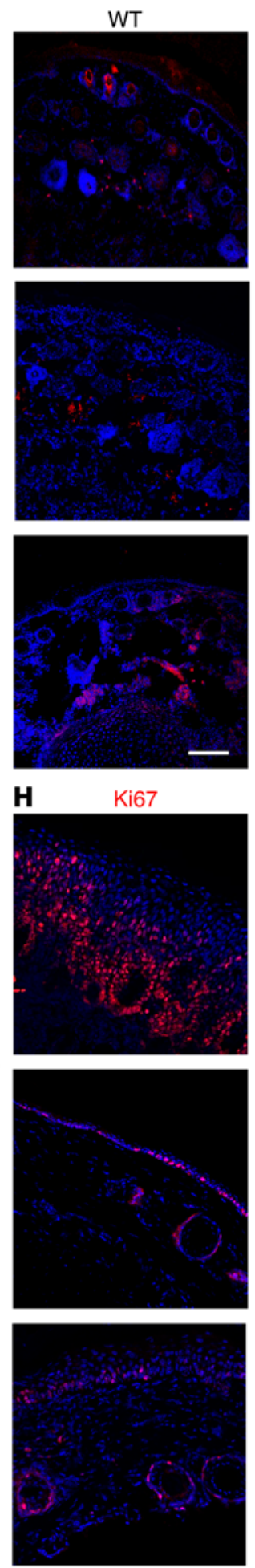

Figure 4. Psoriasiform phenotype in Rac $\mathbf{1}^{\mathbf{V 1 2}}$ mice requires an intact immune system. (A) Flow cytometry showed increased $C D 4^{+}$and $C D 8^{+}$lymphocytes, neutrophils, and DCs in Rac1 $7^{V 12}$ lesional skin. Increased (B) CD3 ${ }^{+}$and (C) ROR $\gamma^{+}$lymphocytes and (D) CD68+ cells (Munro's microabscesses) in the stratum corneum. (E) NOD/SCID Rac7 112 backcrossing resulted in a marked reduction in epidermal thickness and (H) suprabasal proliferation (Ki67), despite (F) persistent RAC1 but not (C) RhoA activity and an absence of tail and limb joint abnormalities. CD3, ROR $\gamma$, CD68, RAC1 GTP, RhoA GTP, Ki67, red; DNA, blue. (A) $n=4$. Error bars represent SEM. (B-H) $n=5$ per condition. Scale bars: $50 \mu \mathrm{m}$. 
A

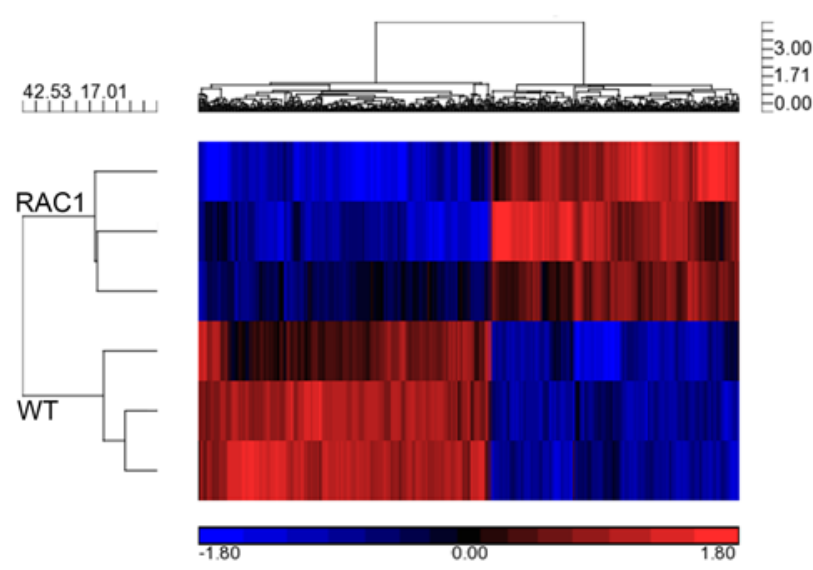

B

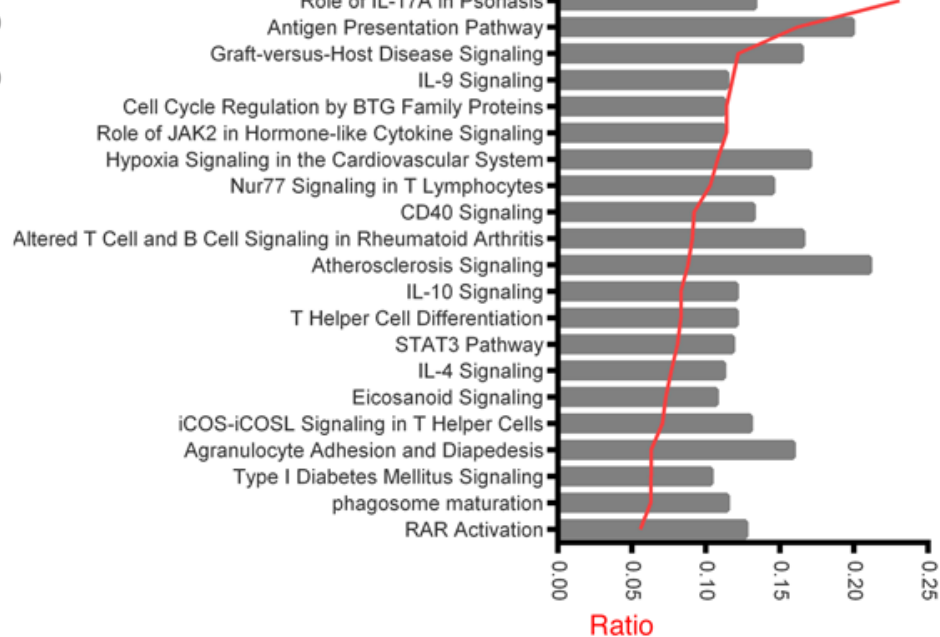

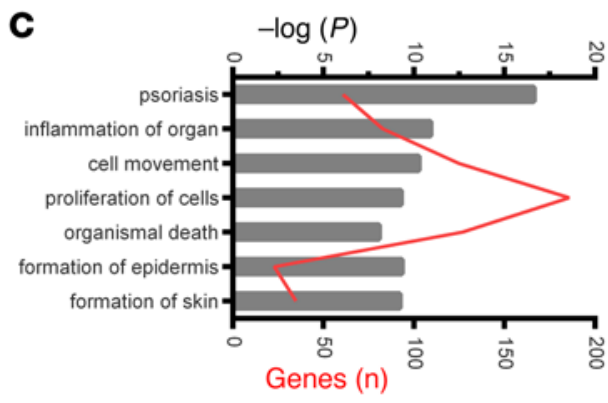

$\mathbf{F}$

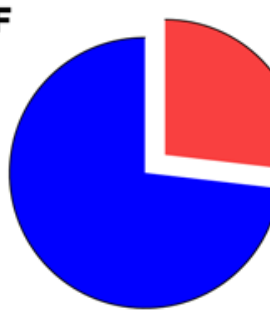

H

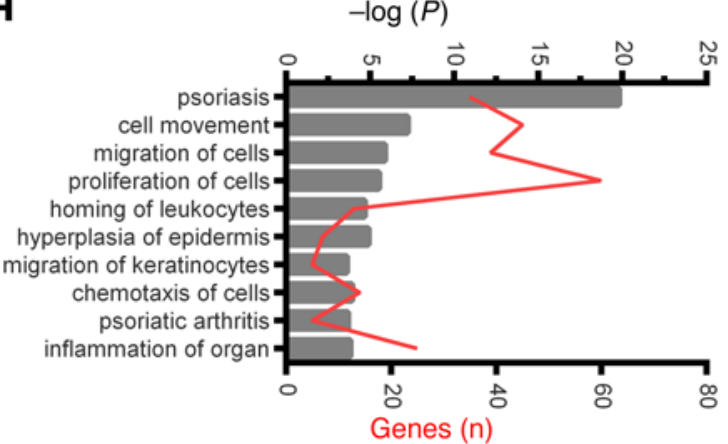

D
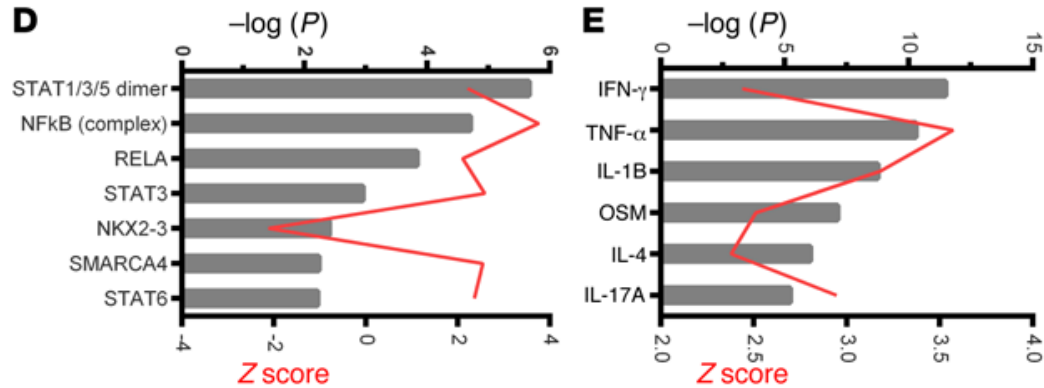

G

$-\log (P)$

Role of IL-17A in Psoriasis IL-10 Signaling

Agranulocyte Adhesion and Diapedesis Graft-versus-Host Disease Signaling Role of Cytokines in Mediating Communication between Immune Cells Granulocyte Adhesion and Diapedesis Protein Ubiquitination Pathway Fatty Acid-oxidation

Hypoxia Signaling in the Cardiovascular System -

Atherosclerosis Signaling Goi Signaling
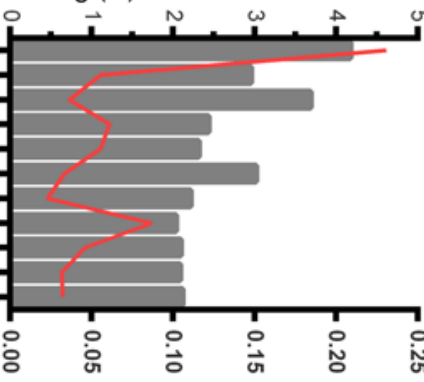

Ratio

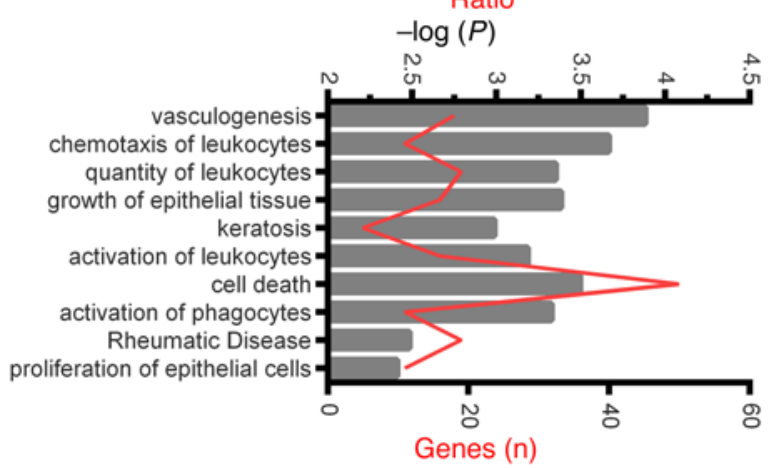

Figure 5. Transcriptional signature of RAC1 activity in human psoriatic skin. (A) Heatmap of DEGs in Rac1 ${ }^{12}$ skin compared with those in littermate control skin, showing that $46 \%$ were upregulated and $54 \%$ were repressed. (B) Canonical pathways (- $\log P$, upper axis, gray; ratio, lower axis, red); (C) biological functions (- $\log P$, upper axis, gray; number of genes, lower axis, red); (D) TFs; and (E) cytokines (-log $P$, upper axis, gray; activation $Z$ score, lower axis, red) of the DEG signature in Rac7 ${ }^{12}$ skin. (F) Overlap $(P<0.05)$ between DEGs in Rac7 12 and human psoriatic skin. (G) Overlap enriched for psoriasis-associated canonical pathways and (H) biological functions. (A-E) $n=3$ per condition; $(\mathbf{F}) n=3$ mice per condition, $n=214$ human psoriasis, and $n=85$ human controls. $P$ values were determined by $(\mathbf{A})$ ANOVA; (F) hypergeometric mean; and (B-E, G, and $\mathbf{H})$ Fisher's exact test. 
A
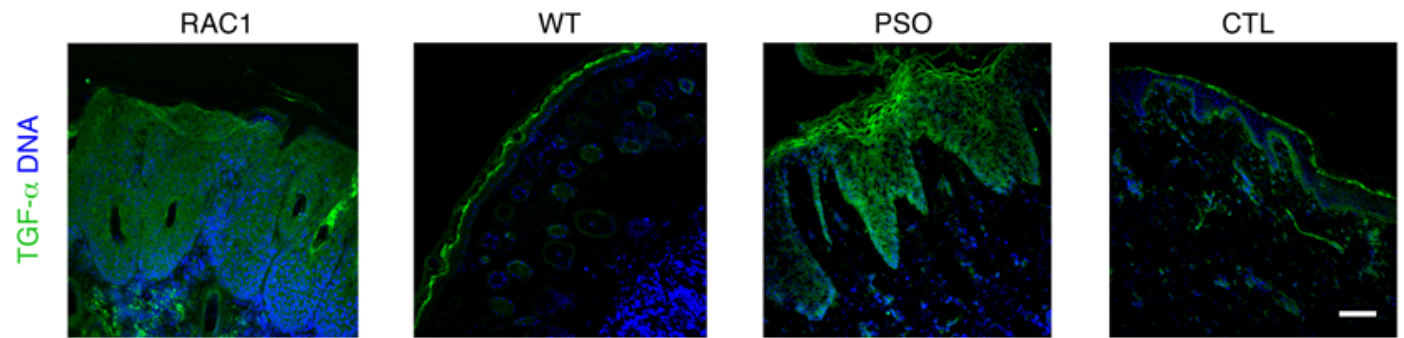

B
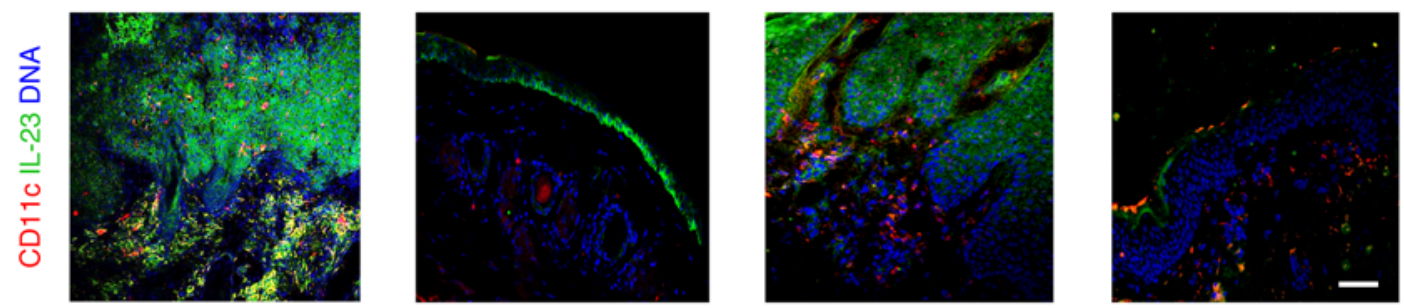

C
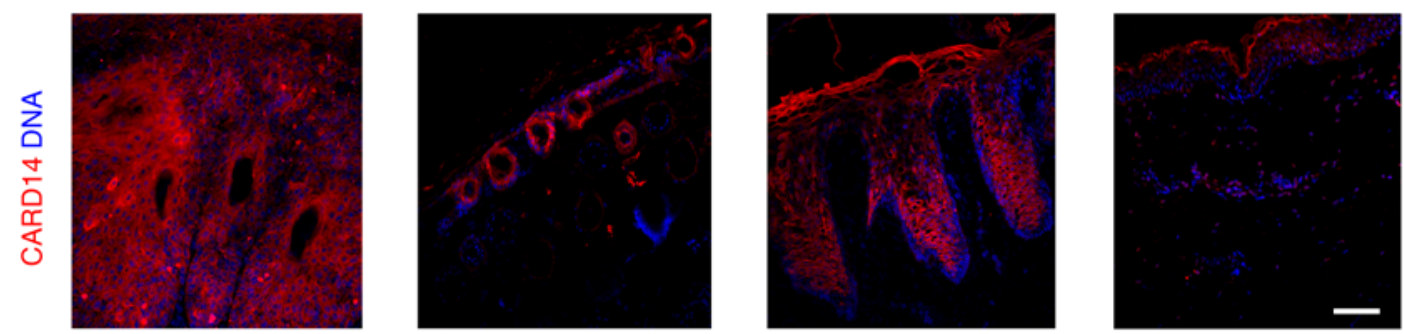

D
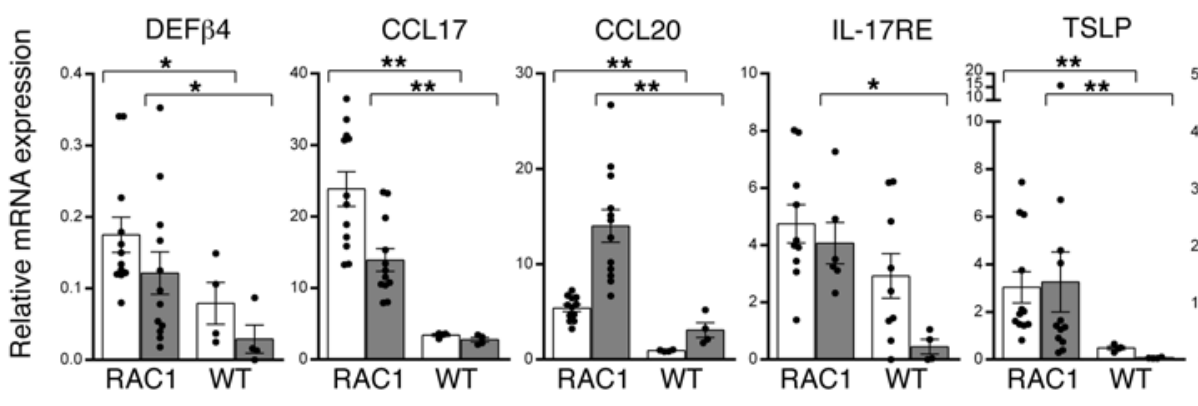

OSMR
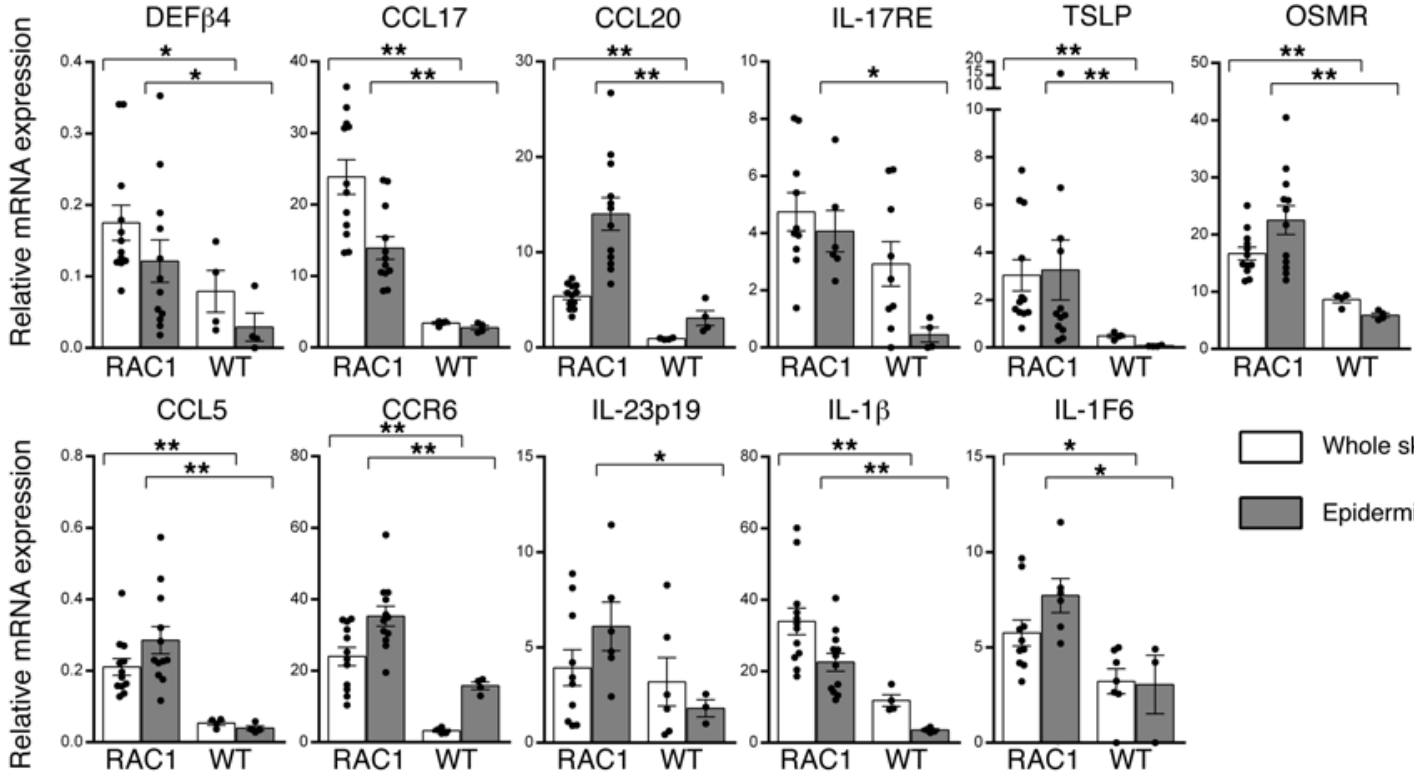

$\mathbf{E}$

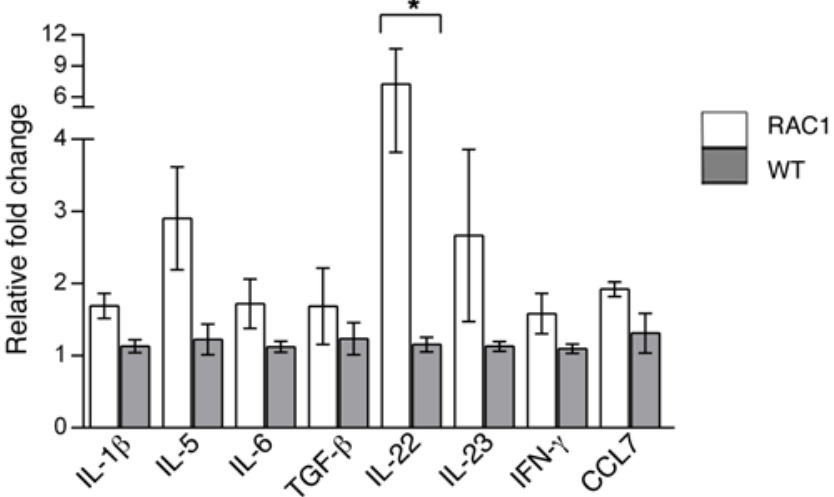

Figure 6. Rac $\mathbf{1}^{V 12}$ mice exhibit differential regulation of the epidermal proteins and cytokines involved in human psoriasis. Expression of (A) TCF- $\alpha$, (B) CD11c and IL-23 p19, and (C) CARD14 in Rac1 ${ }^{12}$, WT, psoriatic lesional, and control skin. TCF- $\alpha$, IL-23p19, green; CD11c, CARD14, red; DNA, blue. (D) RT-qPCR of mRNA from whole skin (white bars) or epidermis (gray bars) from 1-week-old Rac1 ${ }^{1 / 2}$ mice and their age-matched WT littermates, relative to $\beta$-actin. (E) Inflammatory markers in 3-week-old Rac1 ${ }^{12}$ and WT mouse serum. ${ }^{*} P<0.05$ and ${ }^{* *} P<0.005$, by (D) Mann-Whitney $U$ rank sum test and $(\mathbf{E})$ Tukey's multiple comparisons test. Error bars represent SEM. (A-C) $n=5$ per condition; (D) $n=6-12\left(\right.$ Rac $\left.^{112}\right)$ and $n=3-7$ (WT); (E) $n=3$ per condition. Scale bars: $50 \mu \mathrm{m}$. 


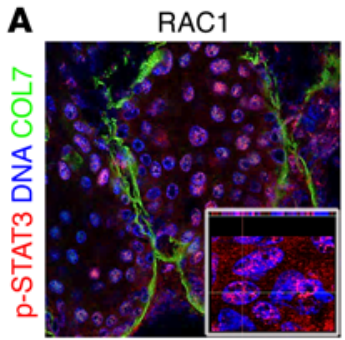

C

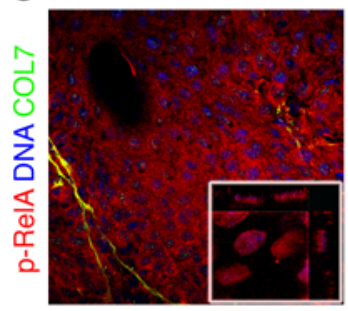

$\mathbf{F}$

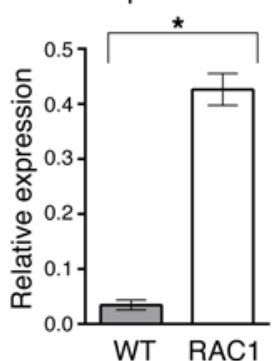

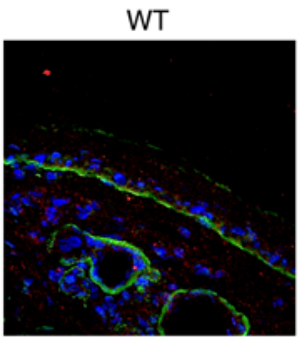

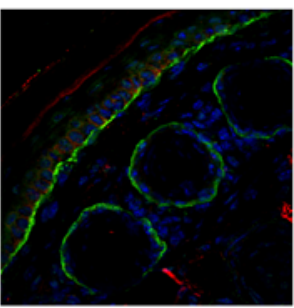

G Acetyl P65

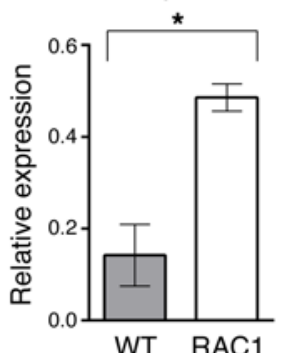

B

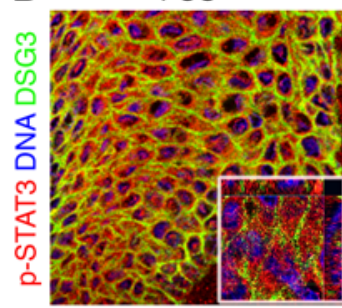

D

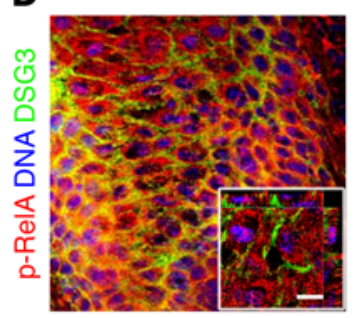

H

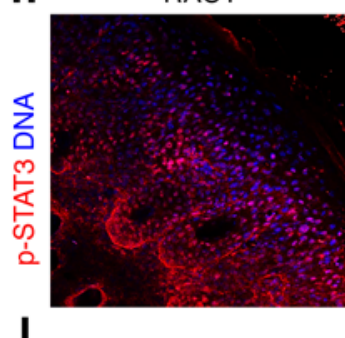

I

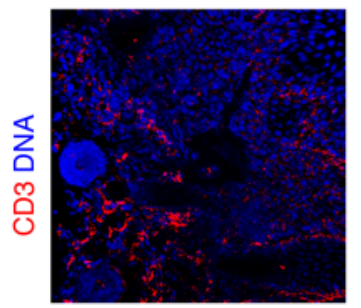

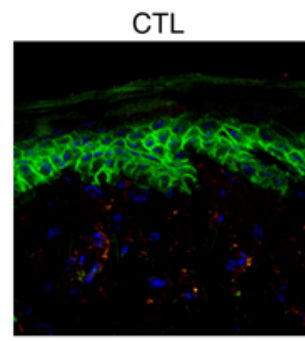

$\mathbf{E}$
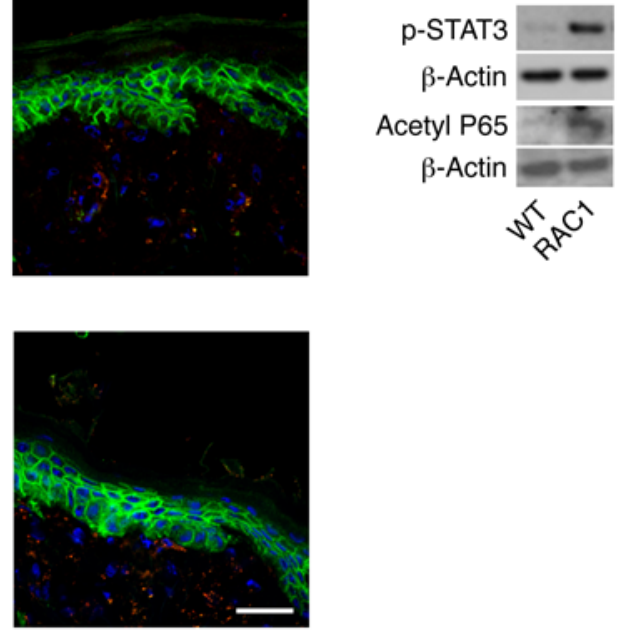

WT
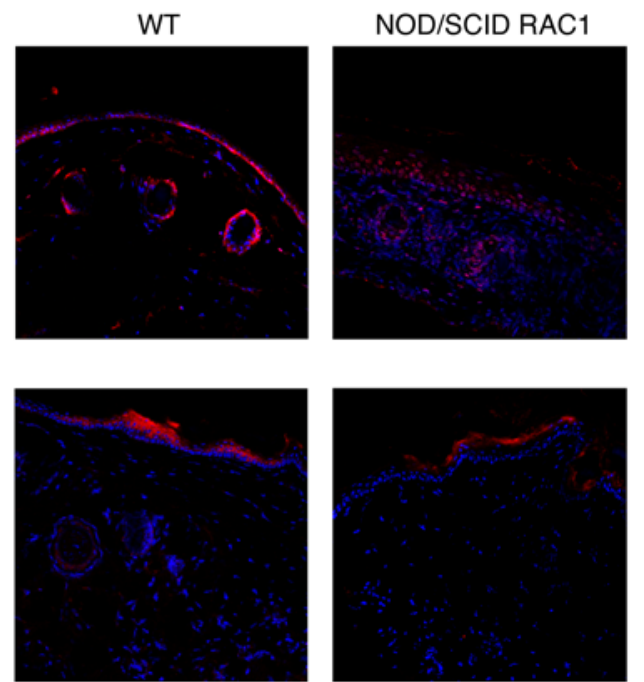

Figure 7. Epidermal RAC1 activation induces NFKB and immune-dependent STAT3 signaling. Expression of epidermal (A and B) p-STAT3, (C and D) p-RELA, and (A-D) COL7 and desmoglein 3 (DSC3), with representative inserts of Z-stacks of Rac $7^{\text {V12 }}$ and psoriasis. p-STAT3, p-RELA, red; COL7, DSC3, green; DNA, blue. (E) Western blot and (F and G) quantification of p-STAT3 and acetyl p65 (relative to $\beta$-actin) in Rac $7^{12}$ and WT skin. Reduced p-STAT3 (H) and CD3 (I) in NOD/SCID Rac1 ${ }^{V 12}$ mice lacking functional lymphocytes. p-STAT3, CD3, red; DNA, blue. Scale bars: $50 \mu \mathrm{m}$ (A-D, H, and I), $10 \mu \mathrm{m}$ (Z-stack inserts $\mathbf{A}-\mathbf{D})$. (A-D) $n=5$ per condition; (E-C) $n=3$ per condition; $(\mathbf{H}$ and $\mathbf{I}) n=3$ per condition. $(\mathbf{F}$ and $\mathbf{G}){ }^{*} P<0.05$, by unpaired $t$ test. Error bars represent SEM. RAC1, Rac1 ${ }^{\text {V12 }}$ mouse.

$\mathrm{NF \kappa B}$ and STAT3 from our bioinformatics analysis for dependence on RAC1 activation. We found that caspase-associated recruitment domain 14 (CARD14) nuclear rim localization and interferon induced with helicase $\mathrm{C}$ domain 1 (IFIH1) nuclear localization were increased in PHKCs compared with that observed in NHKCs; however, expression of a dominant-negative (N17) RAC1 mutant in PHKCs reduced CARD14 and IFIH1 localization. Conversely, expression of RAC1 ${ }^{\text {V12 }}$ in NHKCs increased CARD14 and IFIH1 nuclear rim and nuclear localization, respectively (Figure 8 , A and B). Since our results indicated that immune-derived factors were required for activation of persistent p-STAT3 in Rac1 $1^{\text {V12 }}$ mouse skin, we performed assays to determine how RAC1-activating cytokines (Figure 1, I-K) affect this process. The addition of IL-17A/F increased STAT3 nuclear localization in PHKCs compared with that observed in NHKCs (Figure 8C). Notably, RAC1 $1^{\mathrm{N} 17}$ overexpression led to consistent cytosolic accumulation and loss of nuclear translocation of p-STAT3 in PHKCs (Figure 8C). Conversely $R A C 1^{V 12}$ overexpression in NHKC increased IL-17associated STAT3 nuclear localization (Figure 8C). Immunoblot analysis confirmed increased p-STAT3 in PHKCs compared with that seen in NHKCs (Figure 8, D and E, and Supplemental Figure 9, C and D), which was mimicked by $R A C 1^{\text {V12 }}$ overexpression (Figure 8, D and E), and accumulation of p-STAT3 in $\mathrm{RAC1}^{\mathrm{N17}}$ PHKCs, irrespective of IL-17 stimulation. As the overlapping signature between $R a c 1^{V 12}$ mouse and human psoriatic skin implicates IL-17RC, we verified the presence of this signaling axis in keratinocytes. We detected IL-17RC expression in psoriatic and control keratinocytes (Supplemental Figure 9A) and the IL-17R adaptor TRAF3IP2 (23) in lesional mouse and human psoriatic epidermis (Supplemental Figure 9B). EGF and TNF- $\alpha$ also promoted STAT3 activation (Figure 8, F-I) and nuclear localization (Supplemental Figure 10) in PHKCs, which was reduced follow- 
A

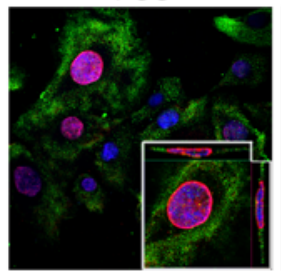

N17

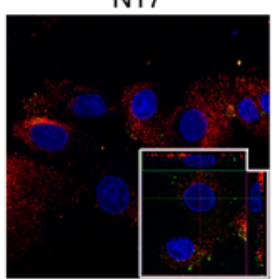

CARD14 RAC1 GTP DNA

D

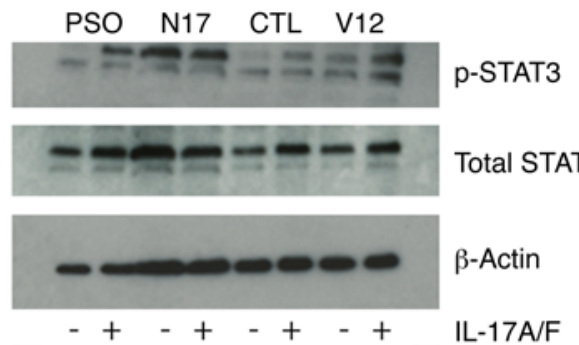

F
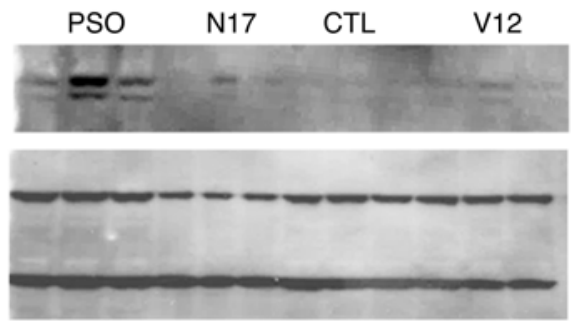

p-STAT3

$\begin{array}{llllllllllll}0 & 10 & 90 & 0 & 10 & 90 & 0 & 10 & 90 & 0 & 10 & 90\end{array}$

G

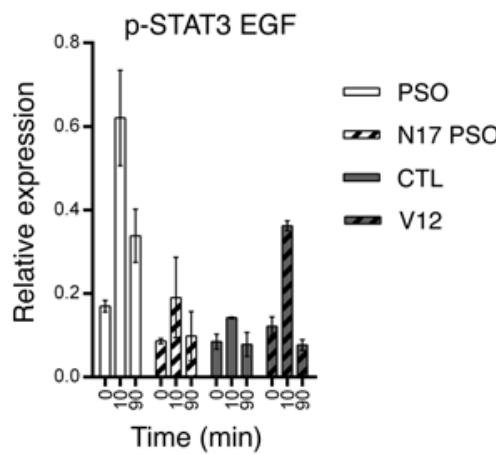

$\beta$-Actin

Time (min)

EGF

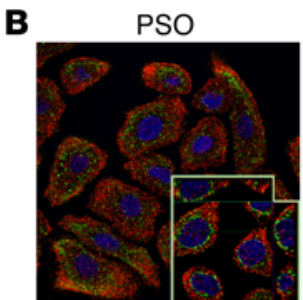

N17

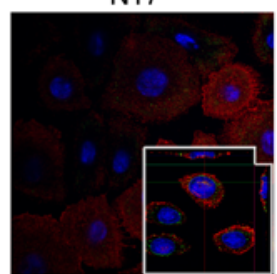

IFIH1 RAC1 GTP DNA

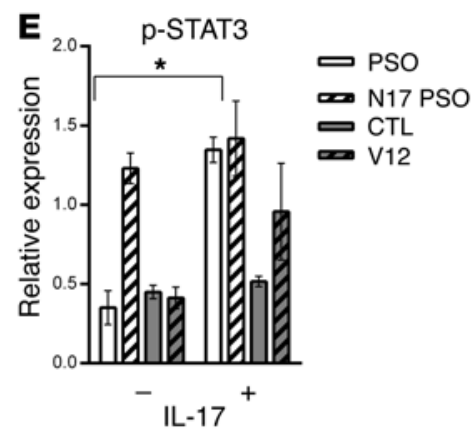

V12

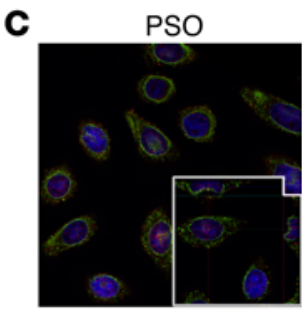

H

H
PSO + IL-17
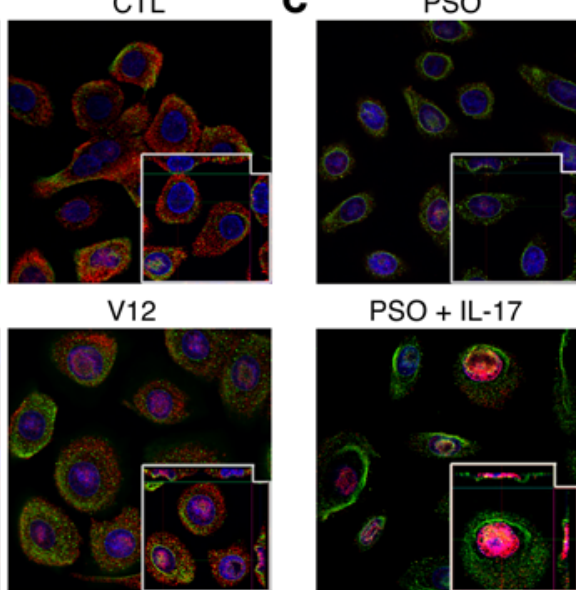

$\mathrm{N} 17+\mathrm{IL}-17$

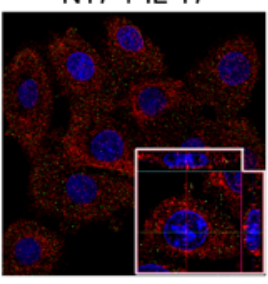

p-STAT3 RAC1 GTP DNA

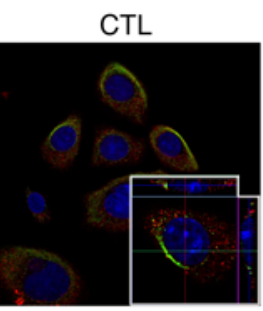

$\mathrm{CTL}+\mathrm{IL}-17$

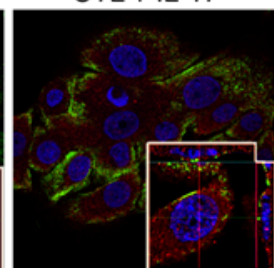

$\mathrm{V} 12+\mathrm{IL}-17$

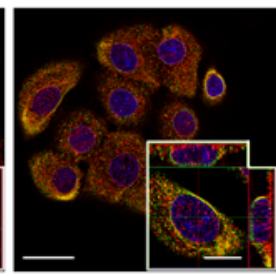

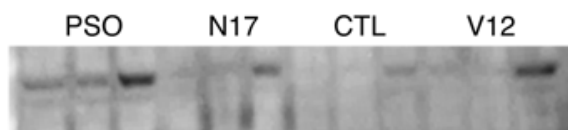

p-STAT3

Total STAT3

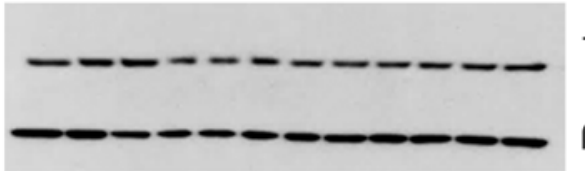

Total STAT3

$\begin{array}{llllllllllll}0 & 10 & 90 & 0 & 10 & 90 & 0 & 10 & 90 & 0 & 10 & 90\end{array}$

$\beta$-Actin

\section{I}

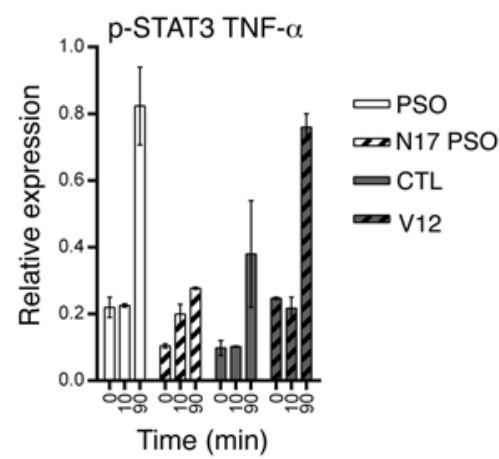

Figure 8. RAC1-dependent signaling in psoriatic keratinocytes of STAT3-, NFKB-, and IFN-related pathways. Expression of (A) CARD14, (B) IFIH1, and

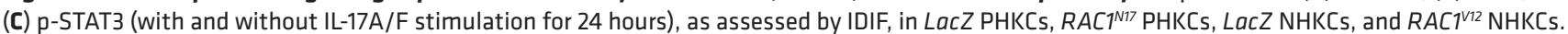
CARD14, IFIH1, p-STAT3, red; RAC1 GTP, green; DNA, blue. (D) Western blot and (E) quantification of p-STAT3 after 0 and 24 hours of IL-17A/F stimulation. ( $\mathbf{F}$ and $\mathbf{H}$ ) Western blots and ( $\mathbf{G}$ and $\mathbf{I})$ quantification of p-STAT3 after 0,10 , and 90 minutes of stimulation with EGF or TNF- $\alpha$. Scale bars: $25 \mu \mathrm{m}(\mathbf{A}-\mathbf{C})$, $10 \mu \mathrm{m} \mathbf{A}-\mathbf{C}$, insert Z-stack). Error bars represent SEM. (E) ${ }^{*} P \leq 0.05$, by unpaired $t$ test. Panels $\mathbf{A}-\mathbf{C}$ are representative images from 3 replicate experiments. (D and $\mathbf{E}) n=3$ per condition; $(\mathbf{F}-\mathbf{I}) n=2$ per condition. 
A

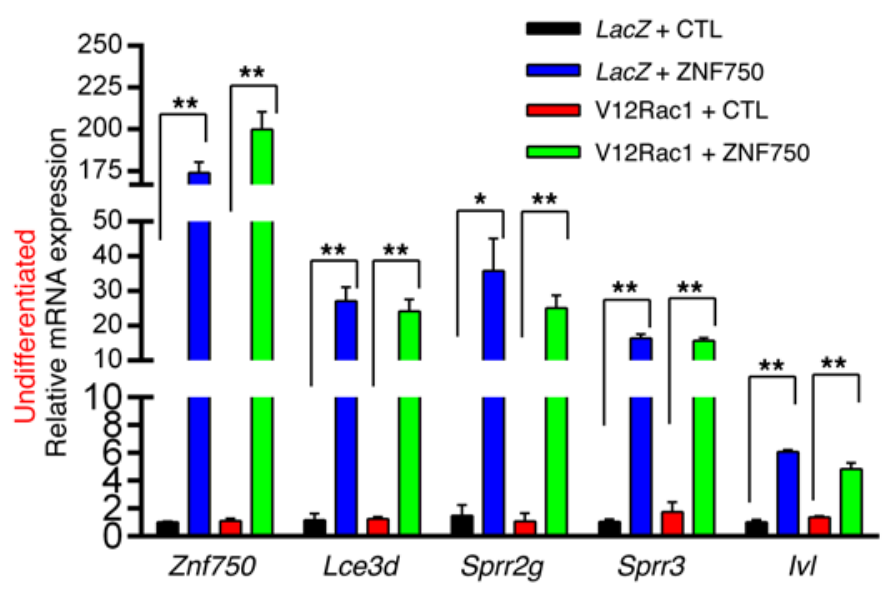

C

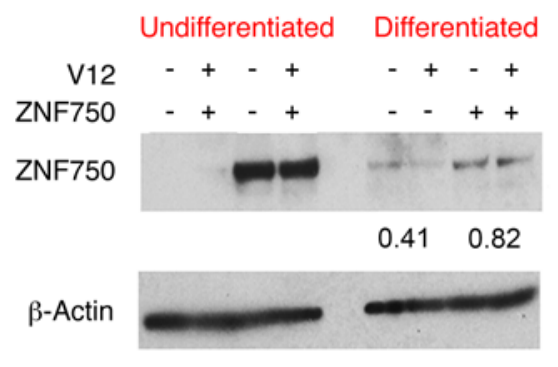

B
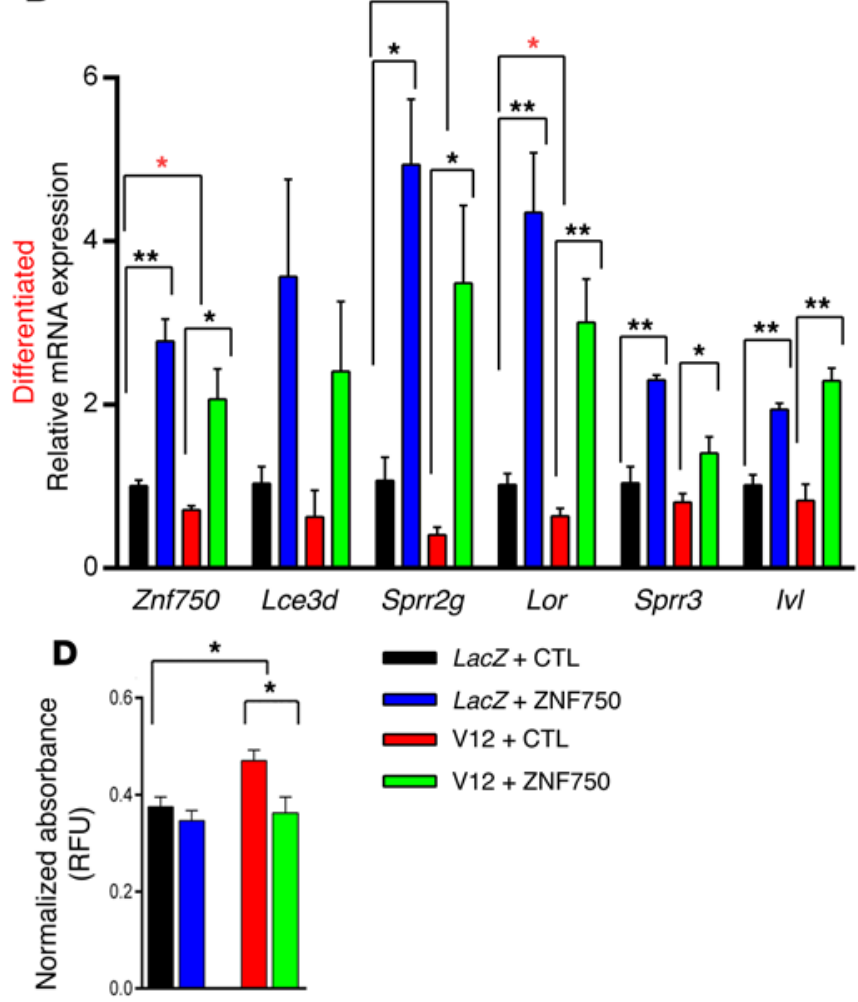

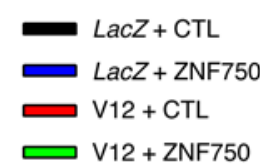

$\mathbf{E}$

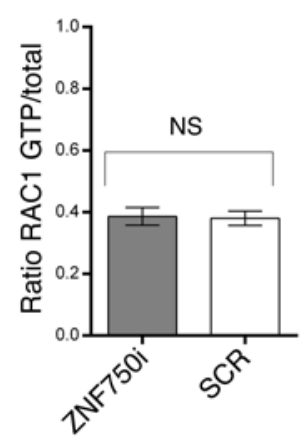

Figure 9. RAC1 drives hyperproliferation and hypodifferentiation through ZNF750. RT-qPCR of mRNA from undifferentiated (A) or differentiated (B) LacZ or Rac7 ${ }^{112}$ NHKCs after ZNF750 or pLEX control overexpression. (C) Protein expression in Rac7 ${ }^{12}$ NHKCs (+) compared with LacZ NHKCs (-) with (+)

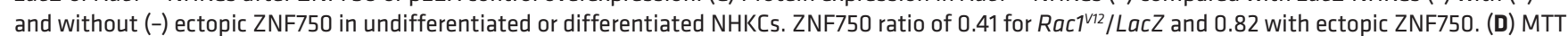
assay of RAC1 ${ }^{12}$ and LacZ NHKCs with ZNF750 or control. RFU, relative fluorescence units. (E) RAC1 GTP pulldown and quantification of siRNA ZNF750 NHKCs compared with scramble siRNA (SCR). (A, B, and E) $n=3$ per condition; (C) $n=2 ;(\mathbf{D}) n=9$ per condition. Error bars represent SEM. ${ }^{*} P<0.05$ and ${ }^{* *} P<0.005$, by 1 -tailed unpaired $t$ test $(\mathbf{A}, \mathbf{B}, \mathbf{D}$, and $\mathbf{E})$.

ing $R A C 1^{N 17}$ overexpression. Conversely, $R A C 1^{V 12}$ overexpression in NHKCs increased STAT3 activation and nuclear localization following $\mathrm{EGF}$ or $\mathrm{TNF}-\alpha$ treatment. We also found a reduced accumulation of p-STAT3 in RAC1 ${ }^{\text {N17 }}$ PHKCs after 24 hours (Figure $8, \mathrm{~F}$ and $\mathrm{H}$ ) compared with that detected after 48 hours (Figure 8D), in agreement with sequestration of cytosolic p-STAT3 without active RAC1. Interestingly, while the effects of EGF on PHKCs and RAC1 ${ }^{\mathrm{V} 12}$ NHKC STAT3 phosphorylation peaked early, at 10 minutes (Figure 8F), suggesting a direct RAC1 effect, the effects of TNF- $\alpha$ on STAT3 phosphorylation were noted only after 90 minutes (Figure 8H) and after 24 hours for IL-17 (Figure 8D), suggesting a delayed or indirect effect. Altogether, this suggests that active RAC1 may regulate these NFאB- and STAT3-associated signaling events in psoriatic keratinocytes.
Given the perturbed differentiation and proliferation in $R a c 1^{V 12}$ murine epidermis in vivo, we compared differentiation markers in RAC1 ${ }^{\text {V12 }}$ NHKCs with those in LacZ NHKCs, including the psoriasis susceptibility gene zinc finger protein 750 (ZNF750) (24). After confirming that ZNF750 upregulated LCE3D, SPRR2G, SPRR3, and IVL mRNA in both RAC1 ${ }^{V 12}$ and LacZ conditions (Figure 9A), we induced differentiation in these cultures. We found modestly reduced ZNF75O mRNA (Figure 9B), but markedly reduced protein (0.42:1) (Figure 9C) in $R A C 1^{\text {V12 }}$ compared with $L a c Z$ NHKCs, accompanied by decreases in mRNA levels of SPRR2G, SPRR3, and LOR. Ectopic ZNF750 restored the $\mathrm{RAC} 1^{\mathrm{v} 12}$-induced reduction to approximately $80 \%$, indicating additional posttranslational downregulation of ZNF750 protein expression. RAC1 ${ }^{12}$ NHKCs showed modestly increased 
A

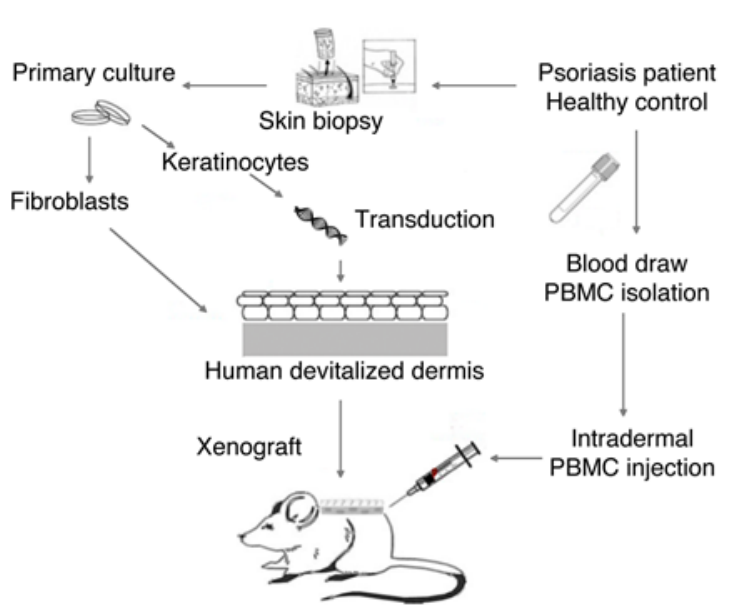

C
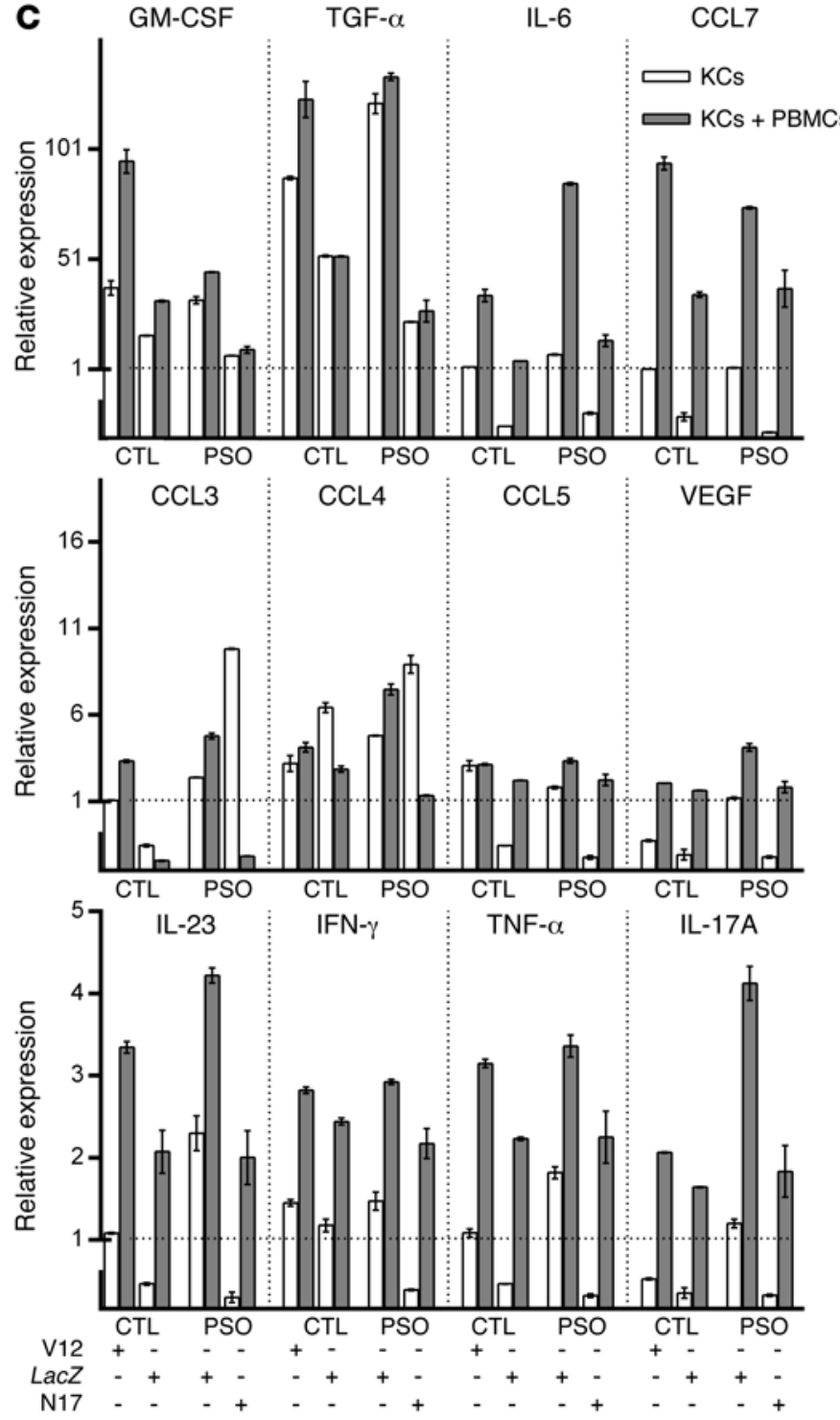

B
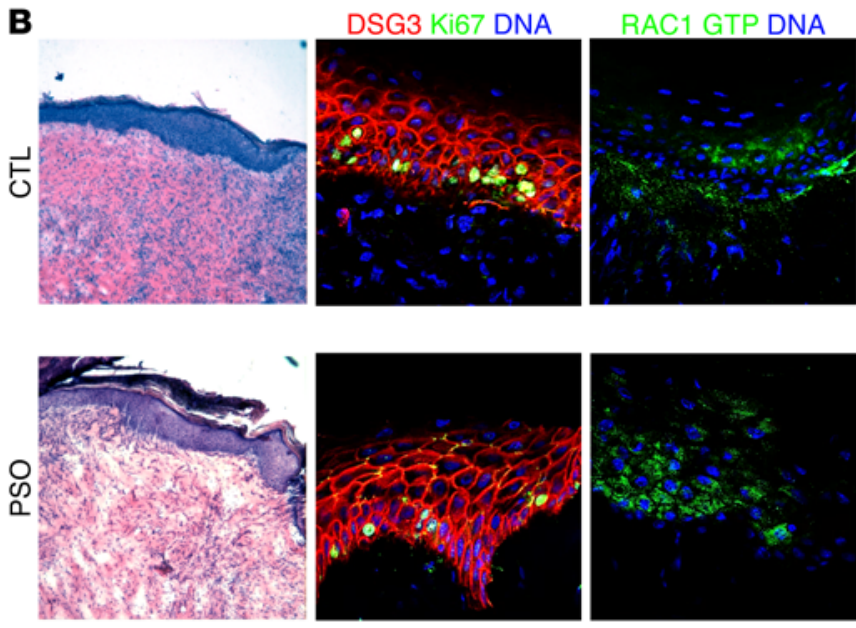
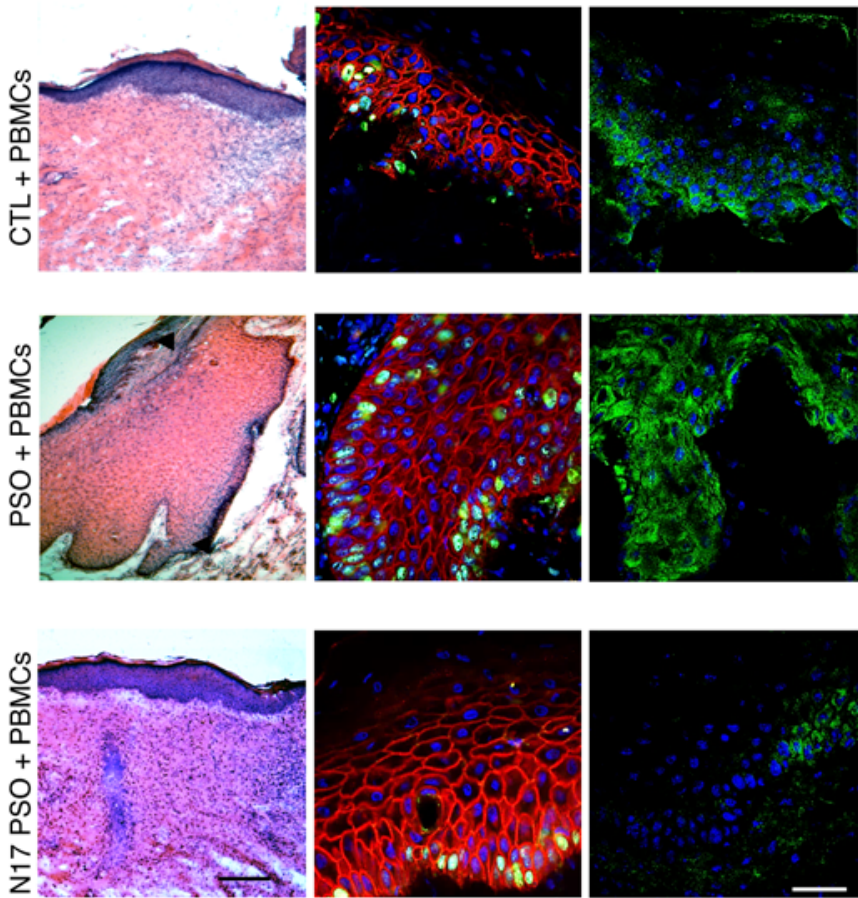

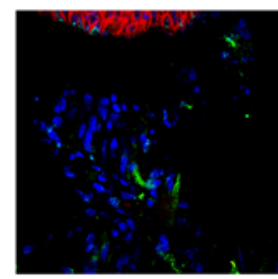

CTL + PBMCs

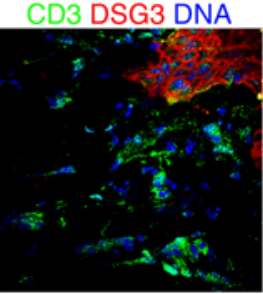

PSO + PBMCs

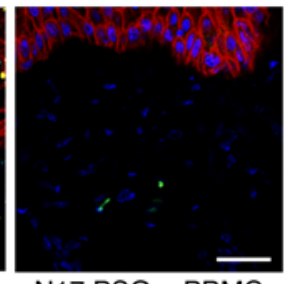

N17 PSO + PBMCs

Figure 10. Epidermal RAC1 promotes an immunoproliferative psoriasis phenotype. (A and B) PHKCs or NHKCs were cultured atop devitalized dermis and xenografted to NOD/SCID mice or after intradermal injection of autologous PBMCs and retroviral keratinocyte-specific transduction of Rac $7^{\mathrm{N} 17}$ or $L a c Z$ control. DSC3, red; RAC1 CTP, Ki67, CD3, green; DNA, blue. (C) Luminex panel of cytokine expression in supernatants of LacZ PHKCs, Rac ${ }^{\mathrm{N17}}$ PHKCs, LacZ NHKCs, and $\operatorname{Rac}^{\mathrm{V} 12}$ NHKCs, alone or in cocultures with PBMCs. Relative expression values were normalized to only PBMCs (dotted line). All conditions were tested in duplicate. (B) $n=2$ per condition. Error bars represent SEM. Scale bars: $100 \mu \mathrm{m}$ (B, left), $50 \mu \mathrm{m}$ (B, middle and right). KCs, keratinocytes; N17, RAC1 ${ }^{\mathrm{N} 17}$. 


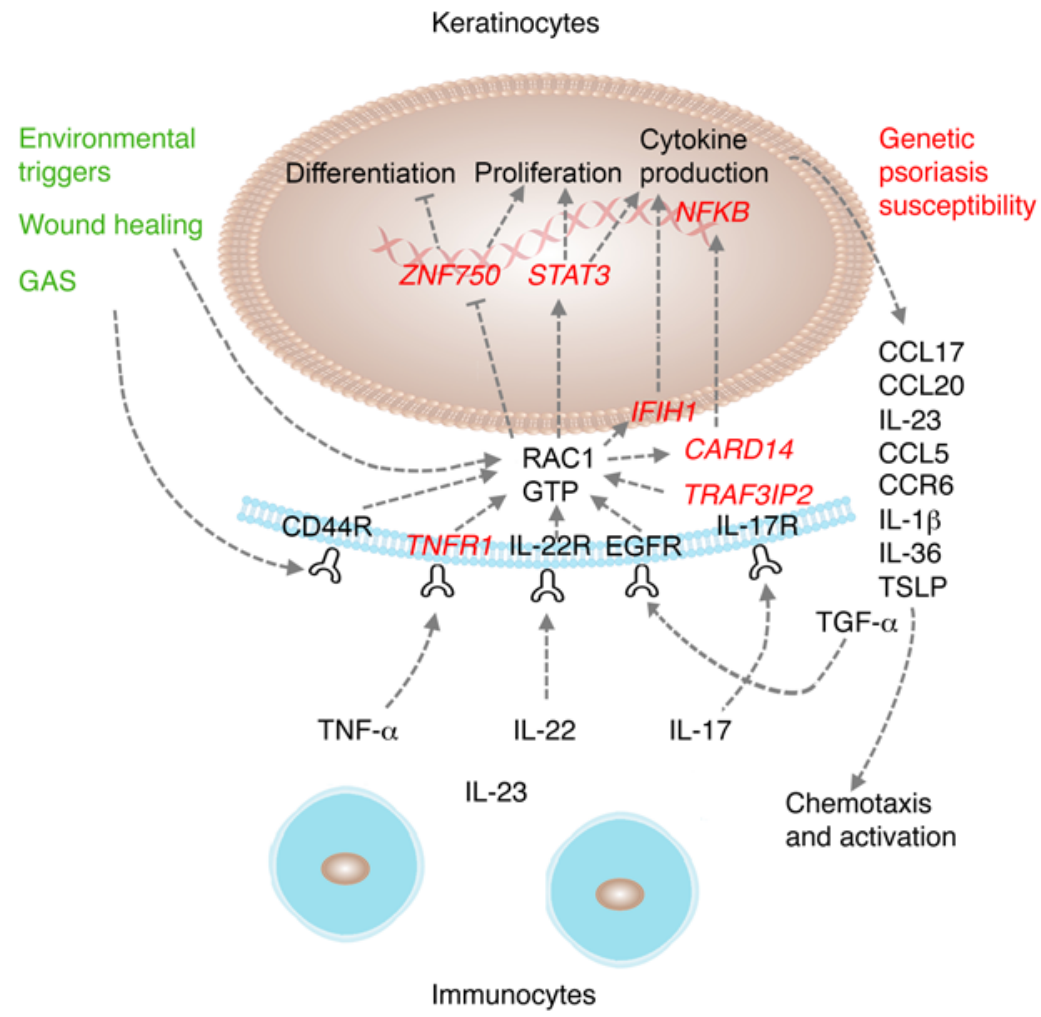

Figure 11. Model of epidermal RAC1 activation driving pathologic epidermis-immune interactions. Schematic model of the potential effect of epidermal RAC1 activation in psoriasis development. Both environmental triggers such as wound healing and CAS (green) and genetic psoriasis susceptibility genes (red) including ZNF750, STAT3, IFIH1, NFKB CARD14, TRAF3IP2, and TNFR1 lie upstream or downstream of RAC1. RAC1 activation by immune-derived factors such as TNF- $\alpha$, IL-17, and IL-22 promotes both proliferation and cytokine production, which may either feed back in an autocrine manner to the epidermis or further promote immune cell chemotaxis and differentiation. proliferation compared with LacZ NHKCs, but ZNF750 induction abolished this difference (Figure 9D). RAC1 GTP pulldown of ZNF750-depleted NHKCs did not activate RAC1 compared with scrambled control (Figure 9E), excluding loss of ZNF750 as an activator of RAC1. These findings demonstrate 1 pathway whereby human keratinocyte activation of RAC1 may inhibit differentiation pathways and promote proliferation through repression of ZNF750 transcription.

PHKCs cultured atop devitalized dermis and xenografted to NOD/SCID mice showed epidermal thickness comparable to that of control NHKC xenografts; however, striking psoriasiform hyperplasia and inflammatory infiltrates were noted in PHKC, but not NHKC, xenografts following the injection of autologous peripheral blood mononuclear cells (PBMCs) (Figure 10, A and B). Hyperplasia and infiltrates in PHKC xenografts were completely normalized following epidermal $R A C 1^{N 17}$ overexpression. To evaluate the role of epidermal RAC1 in promoting inflammation, PHKCs and NHKCs were cocultured in vitro with PBMCs, and cytokines in conditioned medium were assayed after 48 hours. PHKCs and RAC1 $1^{\text {V12 }}$ NHKC cocultures demonstrated elevated expression of an array of cytokines (including granulocyte macrophage-CSF [GM-CSF], TGF- $\alpha$, IL-6, CCL17, CCL3, CCL4, CCL5, VEGF, IL-23, IFN- $\gamma$, TNF- $\alpha$, and IL-17) not seen in $R A C 1^{N 17} \mathrm{PHKC}$ or NHKC cocultures (Figure 10C and Supplemental Figure 11). In total, these results suggest that epidermal RAC1 signaling dictates both proliferative and immune-related aspects of the psoriatic phenotype, but requires both intrinsic activation and immune-derived factors. A model explaining our findings suggests that RAC1 activation lies at the interface of a number of signaling pathways involving genetic psoriasis susceptibility loci (Figure 11).

\section{Discussion}

The central role of epidermal RAC1 in psoriatic epidermis, as summarized in Figure 11, suggests a close association with both environmental triggers as well as genetic psoriasis susceptibility factors. Through its effects on the key TFs STAT3, ZNF750, IRFs, and NFкB, RAC1 appears to inhibit epidermal differentiation, as well as promote epidermal proliferation and production of proliferative and proinflammatory molecules. Some of these secreted agents such as TGF- $\alpha$ may act in an autocrine manner on keratinocyte receptors; however, other proinflammatory agents induced by epidermal RAC1 activation probably promote both immune chemotaxis and differentiation, leading to increased local immune production of TNF- $\alpha$, IL-23, IL-22, and IL-17. Through the ability of TNF- $\alpha$, IL-17, and IL-22 to promote further RAC1 activation, and the ability of RAC1 activation to induce proinflammatory cytokines, epidermal RAC1 activation appears to drive a positive feedback loop between the epidermis and the immune system in promoting psoriasis pathogenesis.

Though epidermal RAC1 hyperactivation was common to human psoriasis, it was not seen in many other epidermal proliferative or inflammatory conditions we studied. Moreover, since previous studies of Rac1-null mice showed normal epidermal proliferation (25) and no inhibition of contact dermatitis-associated inflammation (26), the role of RAC1 in epidermal proliferation and inflammation appeared distinct. However, we found reduced levels of the other Rho family GTPase RhoA and, to a lesser extent, CDC42 in Rac1 ${ }^{V 12}$ lesional and $R A C 1^{V 12}$ NHKCs (Supplemental Figure 5). This suggests an intimate relationship of Rho GTPase regulation in skin homeostasis. We also found an enrichment of RhoGDI signaling in human psoriatic skin (Supplemental Figure 7A). Altogether, these findings are in agreement with those of pre- 
vious studies on Rho family mutants $(27,28)$ and could contribute to our findings. Controlled epidermal cytokine production and proliferation in acute wound healing (29) contrasts with uncontrolled cytokine production and proliferation in psoriatic epidermis (30), and, in fact, some have described psoriasis as exaggerated wound healing (31). In a similar manner, controlled RAC1 activation in wound healing (ref. 13 and Supplemental Figure 6A) contrasted with its wide distribution in lesional psoriatic epidermis.

RAC1 hyperactivation in human psoriasis appeared to occur in a cell-autonomous fashion, as nonlesional PHKCs, cultured for 3 passages in the absence of immunocytes, displayed marked RAC1 activation in response to diverse stimuli, including the known psoriasis therapeutic targets TNF- $\alpha$ and IL-17. However, more data to further validate this claim would be important. The hyaluronaterich GAS capsule, known for its ability to evade immune detection (32) and leukocyte phagocytosis (33), and found in the serum of patients with active infections (34), is a strong inducer of epidermal RAC1 activation (14), with psoriatic keratinocytes showing especially high levels (Supplemental Figure 4A). Thus, it is tempting to speculate that GAS capsular antigen derived from the serum or local microbiota (35) could play a role in triggering pathologic epidermal RAC1 activation during psoriasis flares (11). Although downstream effectors of RAC1 have been genetically associated with psoriasis (Figure 11 and Supplemental Figure 7F) and could lead to feedback effects on RAC1 activity, our results open up the possibility that a genetic predisposition to upstream events causing RAC1 activation is present in some of our psoriatic samples. Although we did not find a consistent deregulation in the RAC1 exchange factors ARHGEF6, TIAM1, or RACGAP1 (Supplemental Figure 4, B and C), others may be altered and provide insights into potential upstream signaling events.

Our transgenic Rac1 mouse studies demonstrate that epidermal RAC1 hyperactivation is sufficient to promote disease activity in the skin, nails, and joints that closely mimics human psoriasis clinically and histologically. RAC1 mice demonstrated the Auspitz sign, Koebnerization, a response to cyclosporine and topical steroids, as well as a pattern of arthritis that closely mimicked human psoriasis. Remission in Rac1 mutants following their crossing with immunodeficient mice is consistent with the immune dependency of human psoriasis (36). RAC1-dependent expression of the chemotactic cytokines CCL2O (37), CCL17 (19), and CCL5 (19) and the Th17 differentiation-promoting cytokines IL-23 (38), IL-1 $\beta$ (30), and IL-6 (39) in mouse epidermis (Figure 6D) and/or human keratinocytes (Figure 10C and Supplemental Figure 11) suggests a role for epidermal RAC1 activation in immune recruitment and activation. Although the relative contributions of IL-23 from keratinocytes and immune cell subsets were not compared, our results support the notion of an additional contribution of IL-23 from immune-stimulated psoriatic keratinocytes to the elevated levels of this cytokine seen in psoriatic skin, in agreement with previous studies on human psoriatic keratinocytes and skin (40). In psoriatic skin samples, we found significant enrichment for RAC1 signaling (Supplemental Figure 7A) in mRNAs including KRT6A/16, S100A7/8/9, IL36A/ IL36RN, STAT3, and CGNL1 (Supplemental Table 3) and overlapping pathway enrichment including in local and systemic psoriasis-associated pathways (Figure 5, G and H). Importantly, we demonstrate that sustained RAC1 activation in epithelia can drive systemic manifestations and activate normal immune cells, a finding that may have implications for other disease states implicating autoimmunity. The role of epidermal RAC1 activation in the promotion of an epidermal-immune feedback loop provides an interesting contrast to activating mutations in other GTPases such as Ras, which have been associated with epidermal neoplasms. One key difference between these two processes may be that RAC1 activation requires immune participation to promote proliferation, whereas Ras activation does not.

We found that RAC1-dependent activation of the inflammatory regulator $\mathrm{NF \kappa B}$ was seen in both $\mathrm{RAC1} 1^{\mathrm{V} 12}$ mouse epidermis and human psoriatic epidermis cells. The psoriasis-associated $\mathrm{NF \kappa B}$ activator CARD14 (17) was upregulated in Rac1 $^{\text {V12 }}$ mouse lesions. RAC1-dependent localization of CARD14 and IFIH1 was also demonstrated in PHKCs. IFIH1, previously implicated in psoriasis GWAS (8), promoted activation of NFאB (41) and IRFs (42). The RAC1-dependent increase in both p-STAT3 and acetylated p65 in mouse skin suggests the involvement of p65 acetylation by STAT3 $(43,44)$, and our backcrossing to immunodeficient mice demonstrates that an intact immune system is required to maintain p-STAT3 in RAC1-activated skin.

The capability of RAC1 to bind (45), activate, and promote nuclear translocation (46) of STAT3 likely explains the RAC1dependent STAT3 activation and nuclear localization seen in $R a c 1^{V 12}$ mouse epidermis and PHKCs. Differences in early and late RAC1-dependent STAT3 activation in PHKCs following EGF, TNF- $\alpha$, and IL-17 treatments, respectively, may be reflective of these multiple ways in which RAC1 can promote STAT3 activity. STAT3 activation has been demonstrated in psoriatic epidermis, and epidermal overexpression of activated STAT3 produced psoriatic skin lesions in mice (47). Interestingly, despite strain and platform discrepancies, we found a significant overlapping DEG signature with the K5STAT3C model (Supplemental Table 4). Both models implicate keratinocyte-intrinsic signaling cascades leading to immunocyte recruitment. The K5-STAT3C and K14AREG mouse models of psoriasis shared pathways enriched for STAT3, immune cell, IL-22, and JAK signaling (Supplemental Figure $7 G$ ). However, unlike $R a c 1^{V 12}$ mouse skin, STAT3 mouse skin required repeated applications of 12-0-teradecanoylphorbol-13-acetate (TPA) or tape stripping to drive lesion development (47). It is possible that, while STAT3 was expressed in the STAT3 mouse, it was only upon injury-induced RAC1 activation (13) that activated RAC1 could effectively transport STAT3 to the nucleus (46), which in turn could have been a key factor driving lesion development.

Our results suggest that activation of RAC1 led to more than a 2-fold reduction in ZNF570 protein levels, which were previously implicated in psoriasis $(48,49)$. Loss of ZNF750 protein expression has been demonstrated to regulate epidermal differentiation and proliferation $(24,50)$ and may be one of several pathways through which RAC1 activation perturbs keratinocyte homeostasis. TGF- $\alpha$ upregulation, previously linked to human psoriatic hyperplasia (51), was another likely effector of RAC1-induced proliferation in mouse lesions. We demonstrate that elevated RAC1 activation is directly linked to an exaggerated proliferation of psoriatic epidermis, but requires an immune-derived stimulus. 
Effective translation of mouse models to in vivo human psoriasis models has proven challenging. Of the 4 transgenic mouse psoriasis models, K5-Tie2, K14-AREG, K5-STAT3, and K5-TGF- $\beta 1$, which were previously studied and correlated with human disease (16), as well as another transgenic psoriasis model of Jun protein deletion (52), none has been extended to a human psoriasis xenograft model. Also, while imiquimod induction of typical psoriasis lesions has been studied in a mouse model (53), a clinical correlation to patients with psoriasis has been questioned $(54,55)$. Our autologous PHKC/PBMC xenograft model (Figure 10, A and B) is, to our knowledge, the first to reproduce the psoriatic hyperplasia and inflammation seen with full-thickness human psoriasis skin/PBMC xenografts (56), while allowing genetic manipulation of epidermal cells prior to xenografting. Epidermal inhibition of RAC1 activation in this model through $R A C 1^{N 17}$ overexpression in PHKCs normalized proliferation and inflammation in treated xenografts of human psoriasis tissue. Thus, the results derived from our in vivo model of human psoriasis correlate well with our observations of human psoriasis tissue and our Rac1 ${ }^{\text {V12 }}$ mouse model. In total, these results suggest that epidermal RAC1 plays a critical role in facilitating the development of a feedback loop between the epidermis and the immune system, promoting both the inflammatory and proliferative phenotypes of psoriasis. Further, we delineate that suppression of immune-derived factors or epidermal RAC1 activation present two distinct pathways for modulating aberrant RAC1 signaling. These findings implicate epidermal RAC1 as a target for psoriasis therapy.

\section{Methods}

Transgenic mice. Rac1 ${ }^{V 12}$-transgenic mice were generated by injection of a Rac1 cDNA construct into the pronucleus of fertilized oocytes. The protocol for cDNA construction and preparation and the screening primers are described in the Supplemental Methods. The founder mouse strain (CBA/CaJ; The Jackson Laboratory) was backcrossed to both C57Bl6 and BALB/c mice (The Jackson Laboratory) and after 5 generations was found to retain the same psoriatic phenotype as that of the founder strain. The founder mouse strain was also backcrossed to NOD/SCID mice (NOD.CB17-Prkdc ${ }^{\text {SCID } / J ~ m i c e ; ~ s t o c k ~ 001303 ; ~ T h e ~}$ Jackson Laboratory) for 3 to 5 generations.

DNFB-induced contact allergic dermatitis and wounding models. For dinitrofluorobenzene-induced (DNFB-induced) contact allergic dermatitis, 1-fluoro-2, 4-dinitrobenzene (Sigma-Aldrich) was diluted in acetone and olive oil ( $4: 1)$. WT mice $(n=3)$ were sensitized by painting $50 \mu \mathrm{l}$ of $0.2 \%$ DNFB on the shaved abdomen on 2 consecutive days. Control mice $(n=3)$ were treated with $50 \mu \mathrm{l}$ acetone and olive oil. For elicitation of contact allergic dermatitis, ears of mice were painted with $10 \mu \mathrm{l}$ of $0.3 \%$ DNFB 10 days later and harvested 24 hours after being painted. For wounding assays, 4-mm punch biopsies harvested from ears were embedded in OCT, and $7-\mu \mathrm{m}$ cryosections were analyzed 24, 48, and 72 hours after wounding.

Cyclosporin A injections. Seven-day-old Rac ${ }^{V 12}$ pups were treated daily i.p. with cyclosporine $(15 \mathrm{mg} / \mathrm{kg}, n=3)$ or vehicle $(n=3)$ for 21 days. Tail sections were harvested and embedded, and 7- $\mu \mathrm{m}$ cryosections were fixed and stained. The average epidermal thickness (excluding the stratum corneum) was measured across 4 different $10 \times$ fields. Ki67 and $\mathrm{CD}^{+}$cells were quantified using ImageJ software (NIH).
Joint imaging. Six-week-old $R a c 1^{V 12}$ mice and their WT littermates $(n=6)$ were placed under isoflurane anesthesia and scanned with the Gamma Medica eXplore CT-120 microCT Scanner (GE Healthcare) at the Stanford small animal imaging facility. The images were taken at $97-\mu \mathrm{m}$ thickness and calibrated, and 3D reconstructions were created using GE Microview software.

$R N A$ extraction and RT-qPCR. Quantitative reverse transcription PCR (RT-qPCR) was performed using the Roche LightCycler 480 with Maxima SYBR Green Master Mix (Fermentas) or SYBR Select Master Mix (Invitrogen). Samples were run in triplicate and normalized to Rn18s RNA. RNA isolation procedures and primer sequences are listed in the Supplemental Material.

Confocal microscopy. Tissue and cell processing is described in the Supplemental Methods. Slides were imaged using a confocal microscope (LSM-700; Zeiss) and processed and quantified using ImageJ. The primary Abs used are listed in the Supplemental Methods.

Immunoblot and pulldown assays. Tissue and cells were lysed in $1 \times$ cell lysis solution (Thermo Fisher Scientific) with 1\% Halt Proteinase-Phosphatase Inhibitor (Thermo Fisher Scientific). The primary Abs used are listed in the Supplemental Methods. For RAC1 GTP pulldown, the ratio of active and total RAC1, respectively, was quantified using an Active RAC1 Pull-Down and Detection Kit (Thermo Fisher Scientific), according to the manufacturer's recommendations. Quantification of immunoblots was performed by densitometry in ImageJ.

Genome-wide transcriptional analysis. Whole skin samples from 7-day-old $\mathrm{Racl}^{\mathrm{V12}}$ pups or normal skin samples from WT littermates $(n=6)$ were analyzed using Illumina MEEBO arrays (NCBI Gene Expression Omnibus [GEO] GSE71683) and compared with a data set of 214 lesional psoriatic skin samples and 85 nonpsoriatic skin samples (GEO GSE13355, GSE14905, GSE30999, and GSE41664), or were compared with gene expression data sets of skin from the K5STAT3C, K14AREG, K5Tie2, K5-TGF- $\beta 1$, and IMQ mouse models of psoriasis (GEO GSE27628). A detailed description of the methodology, the downloaded data sets, and the MIAME-compliant data deposition identification numbers are provided in the Supplemental Methods.

Luminex assays. For human cells, RAC1 ${ }^{V 12}$ or LacZ-overexpressing NHKCs, primary human keratinocytes from nonlesional psoriatic skin retrovirally overexpressing dominant-negative RAC1 (N17) or LacZ (paired), or PBMCs from healthy donors (derived from the Stanford Blood Center and isolated from buffy coats according to standard procedures) were plated in equal density on collagen-coated 6-well plates. After 48 hours in $2 \mathrm{ml}$ keratinocyte growth medium (KGM), either alone (80,000 keratinocytes) or for each keratinocyte condition in coculture with PBMCs (1:10 ratio), supernatants were centrifuged at $228 \times g$ to pellet residual cells. Samples were run in duplicate. For mouse serum analysis, serum was harvested from 3-week-old Rac1 ${ }^{V 12}$ $(n=3)$ or WT littermate $(n=3)$ mice and isolated by centrifugation. Human 51-plex or mouse 38-plex Luminex assays were performed in the Human Immune Monitoring Center at Stanford University. A detailed description is provided in the Supplemental Methods.

Human xenografts. Primary keratinocytes and fibroblasts were isolated from 4-mm punch biopsies of human control or human psoriatic nonlesional skin through dispase treatment (Thermo Fisher Scientific; $30 \mathrm{U} / \mathrm{ml}, 4^{\circ} \mathrm{C}$ overnight) and trypLE digestion (Invitrogen; 15 min, $37^{\circ} \mathrm{C}$ ); seeded on devitalized dermis; and grown at the air-fluid interphase for 7 days before being xenografted to 8-week-old NOD/ 
SCID male mice (The Jackson Laboratory). Autologous PBMCs were injected intradermally and grafts harvested after 14 days. The procedure is described in detail in the Supplemental Methods.

Statistics. Analysis was performed on tissue from 19 psoriasis patients and 10 controls, and in vitro and xenograft studies were performed on cells from 2 to 7 psoriasis patients and 3 to 5 controls. For transgenic mice studies, the groups contained 3-12 animals per group. For transgenic mouse studies, mice were randomized, and analysis was performed in a blinded fashion for all experiments. No outliers were removed from the final analysis. In all 3 model systems, the numbers and replicates are outlined in Methods or the figure legends. Two-tailed (unless otherwise specified), unpaired $t$ tests with Welch's correction and Mann-Whitney $U$ and Wilcoxon rank sum tests or Tukey's multiple comparisons tests with correction for multiple testing were performed to compare mean values between experimental groups using GraphPad Prism 6 (GraphPad Software). Differential gene expression was evaluated by ANOVA using Partek Genomics Suite 6.6, and the resulting $P$ values were significance ranked or corrected for multiple hypothesis testing using an FDR or the Benjamini or Bonferroni method with DAVID (Database for Annotation, Visualization and Integrated Discovery; https://david.ncifcrf.gov/) and KEGG (Kyoto Encyclopedia of Genes and Genomes; http://www.genome.jp/kegg/pathway. html) pathway analysis or the PANTHER (Protein ANalysis THrough Evolutionary Relationships) database. Nonsignificant predictions in IPA were filtered using Fisher's exact test. For gene set comparisons between groups, significance was determined using a hypergeometric test. $P$ values of less than 0.05 were considered statistically significant.

Data and materials availability. Primer sequences, cDNA and siRNA constructs, and vector information, as well as MIAME-compliant NCBI GEO accession numbers (which are also provided above in the Genome-wide transcriptional analysis section), are listed in Supplemental Methods.

Study approval. Mouse studies were approved by and conducted in accordance with Stanford University IACUC guidelines (assur- ance no. A3213-01, protocol 10364). Human studies were conducted according to Declaration of Helsinki principles, in agreement with approved human subject protocols of the IRB of Stanford University School of Medicine (protocol 30586). Informed consent was obtained from all human subjects.

\section{Author contributions}

MCGW performed in vitro, animal, and xenograft experiments, data analysis, statistical analysis, and revised the manuscript. BO, $\mathrm{EW}$, and CND generated transgenic mice and assisted with animal experiments. $\mathrm{LMB}, \mathrm{AKT}, \mathrm{TM}, \mathrm{MD}$, and $\mathrm{AK}$ assisted with in vitro experiments. NTN assisted with in vitro and animal experiments. WL and NEC assisted with animal experiments. DW assisted with data analysis. AWA and MPM collected patients' samples. DS, TH, $\mathrm{BH}, \mathrm{PAK}$, and $\mathrm{MB}$ revised the manuscript and assisted with the direction of the study. MPM conceived the science, oversaw and designed the studies, analyzed the data, and wrote the manuscript.

\section{Acknowledgments}

The authors gratefully acknowledge L. Zaba, E. Engleman, P. Utz, M. Wong, D. Fiorentino, M. Genovese, A. Oro, and L. Benjamin for valuable discussions. This work was supported by grants from the U.S. Department of Veterans Affairs Office of Research and Development; the National Institute of Arthritis and Musculoskeletal and Skin Diseases (NIH grant AR47223, to M.P. Marinkovich); the National Psoriasis Foundation (to M.P. Marinkovich); the European Union's Seventh Framework Programme FP7/2007-2013 (261366, to B. Homey); and the Swedish Society of Medical Research (SSMF), the Swedish Society of Medicine (SLS), and the Fernström Foundation (to M.C.G. Winge).

Address correspondence to: M. Peter Marinkovich, 269 Campus Drive, Rm 2145a, Stanford, California 94305, USA. Phone: 650.498.5425; E-mail:mpm@stanford.edu.
1. Gutowska-Owsiak D, Ogg GS. The epidermis as an adjuvant. J Invest Dermatol. 2012; 132(3 pt 2):940-948.

2. Rachakonda TD, Schupp CW, Armstrong AW. Psoriasis prevalence among adults in the United States. J Am Acad Dermatol. 2014;70(3):512-516.

3. Boehncke WH, Schön MP. Psoriasis. Lancet. 2015;386(9997):983-994.

4. Armstrong EJ, Harskamp CT, Armstrong AW. Psoriasis and major adverse cardiovascular events: a systematic review and meta-analysis of observational studies. J Am Heart Assoc. 2013;2(2):e000062.

5. Viguier M, et al. Efficacy and safety of biologics in erythrodermic psoriasis: a multicentre, retrospective study. Br J Dermatol. 2012;167(2):417-423.

6. Singh JA, et al. Adverse effects of biologics: a network meta-analysis and Cochrane overview. Cochrane Database Syst. 2011;(2):CD008794.

7. Nast A, Jacobs A, Rosumeck S, Werner RN. Efficacy and safety of systemic long-term treatments for moderate-to-severe psoriasis: a systematic review and meta-analysis. J Invest Dermatol. 2015;135(11):2641-2648.

8. Tsoi LC, et al. Identification of 15 new psoriasis susceptibility loci highlights the role of innate immunity. Nat Genet. 2012;44(12):1341-1348.

9. Ryan C, et al. Research gaps in psoriasis: opportunities for future studies. J Am Acad Dermatol. 2014;70(1):146-167.

10. Weiss G, Shemer A, Trau H. The Koebner phenomenon: review of the literature. JEur Acad Dermatol Venereol. 2002;16(3):241-248.

11. Telfer NR, Chalmers RJ, Whale K, Colman G. The role of streptococcal infection in the initiation of guttate psoriasis. Arch Dermatol. 1992;128(1):39-42.

12. Russell AJ, et al. $\alpha 6 \beta 4$ Integrin regulates keratinocyte chemotaxis through differential GTPase activation and antagonism of $\alpha 3 \beta 1$ integrin. J Cell Sci. 2003;116(pt 17):3543-3556.

13. DiPersio CM. Double duty for Rac1 in epidermal wound healing. Sci STKE. 2007;2007(391):pe33.

14. Cywes C, Wessels MR. Group A Streptococcus tissue invasion by CD44-mediated cell signalling. Nature. 2001;414(6864):648-652.

15. Kjoller L, Hall A. Rac mediates cytoskeletal rearrangements and increased cell motility induced by urokinase-type plasminogen activator receptor binding to vitronectin. J Cell Biol.
2001;152(6):1145-1157.

16. Swindell WR, et al. Genome-wide expression profiling of five mouse models identifies similarities and differences with human psoriasis. PLoS One. 2011;6(4):e18266.

17. Jordan CT, et al. Rare and common variants in CARD14, encoding an epidermal regulator of NF-kB, in psoriasis. Am J Hum Genet. 2012;90(5):796-808.

18. Hollox EJ, et al. Psoriasis is associated with increased $\beta$-defensin genomic copy number. Nat Genet. 2008;40(1):23-25.

19. Homey B, Meller S. Chemokines and other mediators as therapeutic targets in psoriasis vulgaris. Clin Dermatol. 2008;26(5):539-545.

20. Volpe E, et al. Thymic stromal lymphopoietin links keratinocytes and dendritic cell-derived IL-23 in patients with psoriasis. J Allergy Clin Immunol. 2014;134(2):373-381.

21. Boniface $\mathrm{K}$, et al. Oncostatin M secreted by skin infiltrating $\mathrm{T}$ lymphocytes is a potent keratinocyte activator involved in skin inflammation. JImmunol. 2007;178(7):4615-4622.

22. Onoufriadis A, et al. Mutations in IL36RN/IL1F5 are associated with the severe episodic inflamma- 
tory skin disease known as generalized pustular psoriasis. Am J Hum Genet. 2011;89(3):432-437.

23. Ellinghaus E, et al. Genome-wide association study identifies a psoriasis susceptibility locus at TRAF3IP2. Nat Genet. 2010;42(11):991-995.

24. Boxer LD, Barajas B, Tao S, Zhang J, Khavari PA. ZNF750 interacts with KLF4 and RCOR1, KDM1A, and CTBP1/2 chromatin regulators to repress epidermal progenitor genes and induce differentiation genes. Genes Dev. 2014;28(18):2013-2026.

25. Chrostek A, et al. Rac1 is crucial for hair follicle integrity but is not essential for maintenance of the epidermis. Mol Cell Biol. 2006;26(18):6957-6970.

26. Pedersen E, et al. RAC1 in keratinocytes regulates crosstalk to immune cells by Arp2/3-dependent control of STAT1. J Cell Sci. 2012;125(pt 22):5379-5390.

27. Garcia-Mata R, Boulter E, Burridge K. The 'invisible hand': regulation of RHO GTPases by RHOGDIs. Nat Rev Mol Cell Biol. 2011;12(8):493-504.

28. Boulter E, et al. Regulation of Rho GTPase crosstalk, degradation and activity by RhoGDI1. Nat Cell Biol. 2010;12(5):477-483.

29. Pastar I, et al. Epithelialization in wound healing: a comprehensive review. Adv Wound Care (New Rochelle). 2014;3(7):445-464.

30. Perera GK, Di Meglio P, Nestle FO. Psoriasis. Annu Rev Pathol. 2012;7:385-422.

31. Haase I, Hobbs RM, Romero MR, Broad S, Watt FM. A role for mitogen-activated protein kinase activation by integrins in the pathogenesis of psoriasis. J Clin Invest. 2001;108(4):527-536.

32. Dinkla K, et al. Upregulation of capsule enables Streptococcus pyogenes to evade immune recognition by antigen-specific antibodies directed to the G-related $\alpha 2$-macroglobulin-binding protein GRAB located on the bacterial surface. Microbes Infect. 2007;9(8):922-931.

33. Leong PA, Cohen MS. Group A streptococcal peptidoglycan-polysaccharide inhibits phagocytic activity of human polymorphonuclear leukocytes. Infect Immun. 1984;45(2):378-383.

34. Gillespie SH, Smith MD, Dickens A, Raynes JG,
McAdam KP. Detection of C-polysaccharide in serum of patients with Streptococcus pneumoniae bacteraemia. JClin Pathol. 1995;48(9):803-806.

35. Zanvit $P$, et al. Antibiotics in neonatal life increase murine susceptibility to experimental psoriasis. Nat Commun. 2015;6:8424.

36. Lowes MA, Suarez-Farinas M, Krueger JG. Immunology of psoriasis. Annu Rev Immunol. 2014;32:227-255.

37. Harper EG, et al. Th17 cytokines stimulate CCL20 expression in keratinocytes in vitro and in vivo: implications for psoriasis pathogenesis. J Invest Dermatol. 2009;129(9):2175-2183.

38. Di Cesare A, Di Meglio P, Nestle FO. The IL-23/ Th17 axis in the immunopathogenesis of psoriasis. J Invest Dermatol. 2009;129(6):1339-1350.

39. Zhou L. IL-6 programs T(H)-17 cell differentiation by promoting sequential engagement of the IL-21 and IL-23 pathways. Nat Immunol. 2007;8(9):967-974.

40. Piskin G, Sylva-Steenland RM, Bos JD, Teunissen MB. In vitro and in situ expression of IL-23 by keratinocytes in healthy skin and psoriasis lesions: enhanced expression in psoriatic skin. JImmunol. 2006;176(3):1908-1915.

41. Mosallanejad K, et al. The DEAH-box RNA helicase DHX15 activates NF-kappaB and MAPK signaling downstream of MAVS during antiviral responses. Sci Signal. 2014;7(323):ra40.

42. Malathi K, Dong B, Gale M Jr, Silverman RH. Small self-RNA generated by RNase L amplifies antiviral innate immunity. Nature. 2007;448(7155):816-819.

43. Frost JA, Swantek JL, Stippec S, Yin MJ, Gaynor $\mathrm{R}$, Cobb MH. Stimulation of NFKB activity by multiple signaling pathways requires PAK1.J Biol Chem. 2000;275(26):19693-19699.

44. Jefferies CA, O'Neill LA. Rac1 regulates interleukin 1-induced nuclear factor $\mathrm{\kappa B}$ activation in an inhibitory protein $\kappa \mathrm{B} \alpha$-independent manner by enhancing the ability of the $\mathrm{p} 65$ subunit to transactivate gene expression. J Biol Chem. 2000;275(5):3114-3120.

45. Simon AR, Vikis HG, Stewart S, Fanburg BL,
Cochran BH, Guan KL. Regulation of STAT3 by direct binding to the Rac1 GTPase. Science. 2000;290(5489):144-147.

46. Faruqi TR, Gomez D, Bustelo XR, Bar-Sagi D, Reich NC. Rac1 mediates STAT3 activation by autocrine IL-6. Proc Natl Acad Sci U S A. 2001;98(16):9014-9019.

47. Sano S, et al. Stat 3 links activated keratinocytes and immunocytes required for development of psoriasis in a novel transgenic mouse model. Nat Med. 2005;11(1):43-49.

48. Yang CF, et al. A promoter sequence variant of ZNF750 is linked with familial psoriasis. J Invest Dermatol. 2008;128(7):1662-1668.

49. Birnbaum RY, et al. Seborrhea-like dermatitis with psoriasiform elements caused by a mutation in ZNF750, encoding a putative $\mathrm{C} 2 \mathrm{H} 2$ zinc finger protein. Nat Genet. 2006;38(7):749-751.

50. Sen GL, et al. ZNF750 Is a p63 target gene that induces KLF4 to drive terminal epidermal differentiation. Dev Cell. 2012;22(3):669-677.

51. Turbitt ML, Akhurst RJ, White SI, MacKie RM. Localization of elevated transforming growth factor-alpha in psoriatic epidermis. J Invest Dermatol. 1990;95(2):229-232.

52. Zenz R, et al. Psoriasis-like skin disease and arthritis caused by inducible epidermal deletion of Jun proteins. Nature. 2005;437(7057):369-375.

53. van der Fits L, et al. Imiquimod-induced psoriasis-like skin inflammation in mice is mediated via the IL-23/IL-17 axis. J Immunol. 2009;182(9):5836-5845.

54. Vinter H, Iversen L, Steiniche T, Kragballe K, Johansen C. Aldara(R)-induced skin inflammation: studies of patients with psoriasis. Br J Dermatol. 2015;172(2):345-353.

55. Mrowietz U. Aldara-induced skin inflammation in mice: close enough to psoriasis? Br J Dermatol. 2015;172(2):313.

56. Boyman O, Hefti HP, Conrad C, Nickoloff BJ, Suter M, Nestle FO. Spontaneous development of psoriasis in a new animal model shows an essential role for resident $\mathrm{T}$ cells and tumor necrosis factor-alpha. JExp Med. 2004;199(5):731-736. 